\title{
On the $\Gamma$-cohomology of rings of numerical polynomials and $E_{\infty}$ structures on $K$-theory
}

\author{
Andrew Baker and Birgit Richter*
}

\begin{abstract}
We investigate $\Gamma$-cohomology of some commutative cooperation algebras $E_{*} E$ associated with certain periodic cohomology theories. For $K U$ and $E(1)$, the Adams summand at a prime $p$, and for $K O$ we show that $\Gamma$-cohomology vanishes above degree 1 . As these cohomology groups are the obstruction groups in the obstruction theory developed by Alan Robinson we deduce that these spectra admit unique $E_{\infty}$ structures. As a consequence we obtain an $E_{\infty}$ structure for the connective Adams summand. For the Johnson-Wilson spectrum $E(n)$ with $n \geqslant 1$ we establish the existence of a unique $E_{\infty}$ structure for its $I_{n}$-adic completion.
\end{abstract}

Mathematics Subject Classification (2000). Primary 55P43, 55N15; Secondary 13D03.

Keywords. Structured ring spectra, $\Gamma$-cohomology, $K$-theory, Johnson-Wilson spectra.

\section{Introduction}

In homotopy theory it is often not sufficient to have homotopy ring structures on a spectrum in order to construct for instance homotopy fixed points under a group action or quotient spectra. For this, it is necessary to have ring structures which are not just given up to homotopy but where these homotopies fulfil certain coherence conditions. We will prove the existence and uniqueness of certain $E_{\infty}$ structures, i.e., structures on spectra which encode a coherent homotopy commutative multiplication.

In [31], [32], Alan Robinson developed a purely algebraic obstruction theory for $E_{\infty}$ structures on homotopy associative and commutative ring spectra. The device for deciding whether a spectrum possesses such a structure is a cohomology theory for commutative algebras, $\Gamma$-cohomology. When applied to the cooperation algebra $E_{*} E$ of a ring spectrum $E$, the vanishing of these cohomology groups implies the existence of an $E_{\infty}$ structure on $E$ which extends the given homotopy ring structure.

\footnotetext{
*The authors would like to thank the Isaac Newton Institute, the Max-Planck-Institut für Mathematik and the Department of Mathematics in Bonn for providing stimulating environments in which this work was carried out and also John Greenlees, Alain Jeanneret, Alan Robinson and Stefan Schwede for helpful comments.
} 
We will apply Robinson's obstruction theory to complex $K$-theory $K U$, its $p$-localization $K U_{(p)}$, its Adams summand $E(1)$, with

$$
K U_{(p)} \simeq \bigvee_{i=0}^{p-2} \Sigma^{2 i} E(1),
$$

and to real $K$-theory $K O$. Our main topological result is

Theorem. There are unique $E_{\infty}$ structures on $K U$, the Adams summand $E(1)$ and $K O$.

This process can then be refined to give $E_{\infty}$ structures on the connective covers. For the higher Johnson-Wilson spectra $E(n)$ with $n \geqslant 1$ we will consider the $I_{n}$-adic completion.

The existence of $E_{\infty}$ structures on $K U$ and $K O$ was already known: $E_{\infty}$ structures for the connective versions $k u$ and $k o$ were constructed in [26, Chapter VIII], and the techniques of [17, VIII] lead to $E_{\infty}$ models for $K U$ and $K O$. Recently, Joachim [22] has described such a structure for $K O$ in the context of symmetric spectra. But as far as we know the uniqueness of these structures has not previously been documented. The existence and uniqueness for $E(1)$ appears to be new. We also show that the connective Adams summand $\ell$ at an odd prime $p$ admits an $E_{\infty}$ structure; on the $p$ completion $\ell_{p}$ an $E_{\infty}$ structure was earlier constructed by McClure and Staffeldt [27]. In subsequent work we have shown that the $E_{\infty}$ structures on $k u, k o, \ell$ and $\ell_{p}$ are unique.

By [18], [29] it is known that the Lubin-Tate spectra $E_{n}$ have unique $E_{\infty}$ structures. In particular, $E_{1}=K U_{p}$ has a unique $E_{\infty}$ structure. The work of Hopkins and Miller [28] and Goerss and Hopkins [18] establishes $A_{\infty}$ and $E_{\infty}$ structures on the Lubin-Tate spectra $E_{n}$ and identified the homotopy type of the space of $A_{\infty}$ (resp. $E_{\infty}$ ) maps between any two of these spectra. With the help of these results the homotopy action of the Morava-stabilizer group on $E_{n}$ could be rigidified to an action of Morava-stabilizer group on $E_{n}$ by $A_{\infty}$ (resp. $\left.E_{\infty}\right)$ maps.

The existence of unique $E_{\infty}$ structures on $K U_{\hat{p}}$ and $E(1) \widehat{p}$ follow directly from the calculation of continuous $\Gamma$-cohomology. In $[19, \S 1]$, Hovey and Strickland asked whether the $I_{n}$-adic completion of $E(n), \widehat{E(n)}$, has $E_{\infty}$ structures.

Theorem. For all $n \geqslant 1$ and all primes $p, \widehat{E(n)}$ possesses a unique $E_{\infty}$ structure.

We give an elementary proof of this which relies on Robinson's obstruction theory [31]; the result for $E_{n}$ then follows using ideas of [34]. However, so far we have not been able to extend this result to $E(n)$ itself since the $\Gamma$-cohomology of $E(n)_{*} E(n)$ appears to be very non-trivial in positive degrees for $n>1$. 
Notation and tools. All otherwise unspecified tensor products are taken over $\mathbb{Z}$ or a localization at a prime $p, \mathbb{Z}_{(p)}$. We denote by $\mathbb{Z}_{p}, \mathbb{Q}_{p}$ and $\widehat{\mathbb{Z}}$ the rings of $p$-adic integers, $p$-adic rationals and profinite integers respectively, while $\widehat{\mathbb{Q}}=\widehat{\mathbb{Z}} \otimes \mathbb{Q}$. For an arbitrary unital commutative ring $R$ we denote the rationalization of $R$ by $R_{\mathbb{Q}}$.

In the body of this paper we will repeatedly use properties of $\Gamma$-cohomology. For the reader's convenience we recall some of its crucial features here.

- Given a commutative $\mathbb{k}$-algebra $A, \Gamma$-cohomology of $A$ with coefficients in an $A$-module $M, \mathrm{H} \Gamma^{*}(A \mid \mathbb{k} ; M)$, is defined in [31] as the cohomology of the derived $A$-homomorphisms from a certain chain complex of $A$-modules $C^{\Gamma}(A \mid \mathbb{k})_{*}$ to $M$.

- This way of defining $\Gamma$-cohomology ensures that each short exact sequence of $A$-modules

$$
0 \rightarrow M^{\prime} \longrightarrow M \longrightarrow M^{\prime \prime} \rightarrow 0
$$

leads to a long exact sequence of $\Gamma$-cohomology groups. It is also clear that in good cases there is a universal coefficient spectral sequence

$$
\mathrm{E}_{2}^{*, *}=\operatorname{Ext}_{A}^{*, *}\left(\mathrm{H} \Gamma_{*}(A \mid \mathbb{k} ; A), M\right) \Longrightarrow \mathrm{H} \Gamma^{*}(A \mid \mathbb{k} ; M) .
$$

Here $\mathrm{H} \Gamma_{*}(A \mid \mathbb{k} ; A)$ denotes the homology of $C^{\Gamma}(A \mid \mathbb{k})_{*}$.

- In $[33,6.8]$, it is shown that $\Gamma$-cohomology vanishes if $A$ is étale over $\mathbb{k}$, and that $\Gamma$-cohomology satisfies Flat Base Change and has a Transitivity Sequence.

- Last but not least, if $A$ is a $\mathbb{k}$-algebra and $\mathbb{Q} \subseteq \mathbb{k}$, then the $\Gamma$-cohomology of $A$ with coefficients in $M$ coincides with André-Quillen cohomology AQ* $(A \mid \mathbb{k} ; M)$; for details see [33, 6.4].

Convenient and concise sources for the definitions of André-Quillen homology and cohomology are [25], [36] where they are denoted $\mathrm{D}_{*}\left(\right.$ ) and $\mathrm{D}^{*}(\mathrm{)}$.

The main techniques we use for the $K$-theoretic examples involve the passage to a continuous version of $\Gamma$-cohomology which we introduce in Section 2, and a description of the $\Gamma$-cohomology groups of the relevant cooperation algebras in terms of $\Gamma$-cohomology groups of colimits of étale algebras at each prime (which turn out to vanish) and $\Gamma$-cohomology groups of algebras which are rationally smooth and can be calculated. At the technical heart of our arguments for the $K$-theoretic examples is Theorem 5.1.

\section{Linear compactness and cohomology}

The results in this section and Section 2 will play a crucial rôle in our proof of Corollary 5.2 when we calculate the $\Gamma$-cohomology of numerical polynomials and more generally for the proof of Theorem 5.1, where Corollary 1.3 and Proposition 1.4 
will help us to identify certain $\Gamma$-cohomology groups of complete algebras with inverse limits of $\Gamma$-cohomology groups of quotient algebras.

Let $\mathbb{k}$ be a commutative Noetherian ring and let $\mathfrak{m} \triangleleft \mathbb{k}$ be a maximal ideal. We topologize $\mathbb{k}$ with respect the $\mathfrak{m}$-adic topology where the open neighbourhoods of 0 are the ideals $\mathfrak{m}^{k} \triangleleft \mathbb{k}$ for $k \geqslant 0$. In the following we will assume that $\mathbb{k}$ is Hausdorff with respect to this $\mathfrak{m}$-adic topology, i.e., that

$$
\bigcap_{k \geqslant 0} \mathfrak{m}^{k}=0
$$

For each $k \geqslant 0, \mathfrak{m}^{k}$ is a finitely-generated $\mathbb{k}$-module, while $\mathfrak{m}^{k} / \mathfrak{m}^{k+1}$ is a finitelygenerated $\mathbb{k} / \mathfrak{m}$-module which is therefore an Artinian $\mathbb{k} / \mathfrak{m}$-module.

Now let $A$ be a commutative unital $\mathbb{k}$-algebra. The ideals $\mathfrak{m}^{k} A=A \mathfrak{m}^{k} \triangleleft A$ also generate a topology on $A$ which is Hausdorff if

$$
\bigcap_{k \geqslant 0} A \mathfrak{m}^{k}=0
$$

Then the unit homomorphism $\mathbb{k} \longrightarrow A$ is automatically continuous and if $A$ is augmented over $\mathbb{k}$ then the augmentation is also continuous. Furthermore, $(A, \mathbb{k})$ is a topological algebra over the topological ring $\mathbb{k}$. We say that $(A, \mathbb{k})$ is Hausdorff if both (1.1) and (1.2) hold.

If $(A, \mathbb{k})$ is a Hausdorff topological algebra, then the $\mathfrak{m}$-adic completion of $(A, \mathbb{k})$ is $\left(\widehat{A_{\mathfrak{m}}}, \widehat{\mathbb{k}_{\mathfrak{m}}}\right)$, where

$$
\widehat{A_{\mathfrak{m}}}=\lim _{k} A / \mathfrak{m}^{k} A, \quad \widehat{\mathbb{k}_{\mathfrak{m}}}=\lim _{k} \mathbb{k} / \mathfrak{m}^{k}
$$

We say that $(A, \mathbb{k})$ is $\mathfrak{m}$-adically complete if $\widehat{A_{\mathfrak{m}}}=A$ and $\widehat{k_{\mathfrak{m}}}=\mathbb{k}$. When $\mathfrak{m}$ is clear from the context we will sometimes simplify notation by writing $(\widehat{A}, \widehat{\mathbb{k}})=\left(A_{\mathfrak{m}}, \widehat{k_{\mathfrak{m}}}\right)$. If $(A, \mathbb{k})$ is augmented over $\mathbb{k}$ then $\left(\widehat{A_{\mathfrak{m}}}, \widehat{\mathbb{k}_{\mathfrak{m}}}\right)$ is augmented over $\mathbb{\mathbb { k } _ { \mathfrak { m } }}$.

Recall from [15], [21], [37] the property of a topological left module $M$ over a topological algebra $(A, \mathbb{k})$ being linearly compact:

- If $\left\{x_{\lambda}+N_{\lambda}\right\}_{\lambda \in \Lambda}$ is a collection of cosets of closed submodules $N_{\lambda} \subseteq M$ such that every finite collection of the cosets $x_{\lambda}+N_{\lambda}$ has non-trivial intersection, then

$$
\bigcap_{\lambda \in \Lambda} x_{\lambda}+N_{\lambda} \neq \varnothing
$$

We will make repeated use of the following vanishing result of [21, p. 57, théorème 7.1] for the higher derived functors of the inverse limit for inverse systems of linearly compact modules. 
Theorem 1.1. Let $\left\{M_{i}\right\}_{i \in I}$ be an inverse system of linearly compact A-modules and continuous A-linear maps. Then for all $s>0$ we have

$$
\lim _{i}^{s} M_{i}=0 .
$$

Recall that a topological $\mathbb{k}$-module $M$ is topologically free on a countable basis $\left\{b_{i}\right\}_{i \geqslant 1}$ if for each element $x \in M$ and $k \geqslant 1$, in $M / \mathfrak{m}^{k} M$ considered as a $\mathbb{k} / \mathfrak{m}^{k}$ module, there is a unique (finite) expansion

$$
\bar{x}=\sum_{i \geqslant 1} \bar{r}_{i} \bar{b}_{i}
$$

with $\bar{r}_{i} \in \mathbb{k} / \mathfrak{m}^{k}$ and where $\bar{b}_{i} \in M / \mathfrak{m}^{k} M$ is the residue class of $b_{i}$. As a consequence, $x$ has a unique expansion as a limit sum

$$
x=\sum_{i \geqslant 1} t_{i} b_{i}
$$

where $t_{i} \rightarrow 0$ as $i \rightarrow \infty$; this means that for each $k$, there is an $n_{k}$ such that for $i>n_{k}$ we have $t_{i} \in \mathfrak{m}^{k}$. The linear topology on $M$ has basic open neighbourhoods of 0 of the form $\mathfrak{m}^{k} M$. Now the Noetherian condition on $\mathbb{k}$ implies that

$$
\mathfrak{m}^{k} M=\left\{\sum_{i \geqslant 1} t_{i} b_{i}: t_{i} \in \mathfrak{m}^{k}, t_{i} \rightarrow 0 \text { as } i \rightarrow \infty\right\} .
$$

For two topological left $R$-modules $L$ and $M$ over a commutative topological ring $R$, we let

$$
\mathscr{H o m}_{R}(L, M) \subseteq \operatorname{Hom}_{R}(L, M)
$$

be the submodule of continuous $R$-module homomorphisms.

Proposition 1.2. Suppose that $M$ is a finitely generated $\mathbb{k}$-module which is complete and Hausdorff with respect to the $\mathfrak{m}$-adic topology. If $L$ is a $\mathbb{k}$-module which is complete with respect to the $\mathfrak{m}$-adic topology and topologically free on a countable basis then $\mathscr{H o m}_{\mathbb{k}}(L, M)$ is a linearly compact $\mathbb{k}$-module.

Proof. Assume first that $L$ is Hausdorff with respect to the $\mathfrak{m}$-adic topology. Note that

$$
\mathscr{H o m}_{\mathbb{k}}(L, M)=\mathscr{H o m}_{\mathbb{k}}\left(L, \lim _{k} M / \mathfrak{m}^{k} M\right)=\lim _{k} \mathscr{H o m}_{\mathbb{k}}\left(L, M / \mathfrak{m}^{k} M\right) .
$$

If $\left\{b_{j}\right\}_{j \geqslant 1}$ is a topological basis for $L$, then using the Noetherian condition on $\mathbb{k}$ we find that the basic neighbourhoods of 0 in $L$ are the submodules $\mathfrak{m}^{k} L \subseteq L$. From 
this we find that

$$
\begin{aligned}
\mathscr{H o m}_{\mathbb{k}}\left(L, M / \mathfrak{m}^{k} M\right) & =\mathcal{H o m}_{\mathbb{k} / \mathfrak{m}^{k}}\left(L / \mathfrak{m}^{k} L, M / \mathfrak{m}^{k} M\right) \\
& =\operatorname{Hom}_{\mathbb{k} / \mathfrak{m}^{k}}\left(L / \mathfrak{m}^{k} L, M / \mathfrak{m}^{k} M\right) \\
& =\prod_{j \geqslant 1} \operatorname{Hom}_{\mathbb{k} / \mathfrak{m}^{k}}\left(\left(\mathbb{k} / \mathfrak{m}^{k}\right) b_{j}, M / \mathfrak{m}^{k} M\right) .
\end{aligned}
$$

But

$$
\operatorname{Hom}_{\mathbb{k} / \mathfrak{m}^{k}}\left(\left(\mathbb{k} / \mathfrak{m}^{k}\right) b_{j}, M / \mathfrak{m}^{k} M\right)=M / \mathfrak{m}^{k} M
$$

and this is Artinian, hence linearly compact. This in turn implies that the final product above is also linearly compact. The claim now follows since $\lim _{k} \mathscr{H}_{\mathrm{K}}\left(L, M / \mathrm{m}_{\mathbb{k}}^{k} M\right)$ is a closed subspace of the product

$$
\prod_{j \geqslant 1} \operatorname{Hom}_{\mathbb{k} / \mathfrak{m}^{k}}\left(\left(\mathbb{k} / \mathfrak{m}^{k}\right) b_{j}, M / \mathfrak{m}^{k} M\right) .
$$

Now we consider what happens when $L$ is not necessarily Hausdorff. In this case, Nakayama's Lemma implies that for any $f \in \mathscr{H o m}_{\mathbb{k}}(L, M)$ we have

$$
f\left(\bigcap_{k \geqslant 1} \mathfrak{m}^{k} L\right)=0
$$

Hence such an $f$ factors through the quotient

$$
L_{0}=L / \bigcap_{k \geqslant 1} \mathfrak{m}^{k} L
$$

so we might as well replace $L$ by this Hausdorff quotient. Then we have

$$
\mathscr{H o m}_{\mathbb{k}}(L, M)=\mathcal{H o m}_{\mathbb{k}}\left(L_{0}, M\right) .
$$

We can apply this to prove the following.

Corollary 1.3. Suppose further that $A$ is a topological $\mathbb{k}$-algebra with respect to the $\mathfrak{m}$-adic topology inherited from $\mathbb{k}$ and that $L$ and $M$ are topological $A$-modules. Then $\mathcal{H o m}_{A}(L, M) \subseteq \mathscr{H}_{\mathbb{k}}(L, M)$ is a closed $\mathbb{k}$-submodule. Hence $\mathscr{H}_{\mathrm{k}}(L, M)$ is linearly compact.

Proof. Again we first consider the case where $L$ is Hausdorff. The two continuous action maps

$$
A \otimes_{\mathbb{k}} \mathcal{H o m}_{\mathbb{k}}(L, M) \longrightarrow \mathcal{H o m}_{\mathbb{k}}(L, M)
$$

given by

$$
a \otimes f \longmapsto a f, \quad a \otimes f \longmapsto f(a(-))
$$

are equalized on $\mathscr{H o m}_{A}(L, M)$, so this is a closed subset of $\mathscr{H o m}_{\mathbb{k}}(L, M)$. For $L$ not Hausdorff we see as above that $\operatorname{Hom}_{A}(L, M) \cong \operatorname{Hom}_{A}\left(L_{0}, M\right)$. 
Note that if $A$ is not necessarily Hausdorff, then setting

$$
A_{0}=A / \bigcap_{k \geqslant 1} \mathfrak{m}^{k} A
$$

we have

$$
\mathscr{H o m}_{A}(L, M)=\mathscr{H o m}_{A_{0}}\left(L_{0}, M\right) .
$$

Proposition 1.4. Let $\left(C^{*}, \delta\right)$ be a cochain complex of linearly compact and Hausdorff $\mathbb{k}$-modules where for each $n$, the coboundary $\delta^{n}: C^{n} \longrightarrow C^{n+1}$ is continuous. Then for each $n, \mathrm{H}^{n}\left(C^{*}, \delta\right)$ is linearly compact.

Proof. Since each $C^{n}$ is linearly compact and Hausdorff, the submodules $\operatorname{Im} \delta^{n-1}$ and $\operatorname{Ker} \delta^{n}$ of $C^{n}$ are both closed. Therefore

$$
\mathrm{H}^{n}\left(C^{*}, \delta\right)=\operatorname{Ker} \delta^{n} / \operatorname{Im} \delta^{n-1}
$$

is also linearly compact.

\section{Continuous $\Gamma$-cohomology}

In this section we discuss some technical issues related to our calculations of $\Gamma$-cohomology later in the paper. Continuous cohomology of profinite groups is described in [35], [36]; for analogues appearing in topology see [7], [10]; our present theory is modelled closely on the presentations in those references.

Let $\mathbb{k}$ be a commutative Noetherian ring and let $\mathfrak{m} \triangleleft \mathbb{k}$ be a maximal ideal. We topologize $\mathbb{k}$ with the $\mathfrak{m}$-adic topology. Let $M$ be a topological left module over a topological algebra $(A, \mathbb{k})$. In practise, we will usually consider the $\mathfrak{m}$-adic topology on $M$.

In the following we shall consider $\Gamma$-cohomology of $A$ with coefficients in $M$, $\mathrm{H} \Gamma^{*}(A \mid \mathbb{k} ; M)$. In [31], [33], $\Gamma$-cohomology is defined using a cochain complex $\operatorname{Hom}_{A}\left(C^{\Gamma}(A \mid \mathbb{k})_{*}, M\right)$. Here $C^{\Gamma}(A \mid \mathbb{k})_{*}$ is the chain complex whose degree $n$-part is the left $A$-module

$$
C^{\Gamma}(A \mid \mathbb{k})_{n}=\bigoplus_{s+r=n} \operatorname{Lie}(s+1)^{*} \otimes \mathbb{k}\left[\Sigma_{s+1}\right]^{\otimes r} \otimes_{\mathbb{k}} A^{\otimes(s+2)},
$$

where $\operatorname{Lie}(s+1)^{*}$ is the $\mathbb{k}$-linear dual of the $(s+1)$-st term of the Lie operad and $\Sigma_{\ell}$ denotes the symmetric group on $\ell$ letters. In particular, $\operatorname{Lie}(s+1)^{*}$ and $\mathbb{k}\left[\Sigma_{s+1}\right]$ are finite-rank free $\mathbb{k}$-modules. Topologising $C^{\Gamma}(A \mid \mathbb{k})_{*}$ with the $\mathfrak{m}$-adic topology, we can introduce the subcomplex

$$
\mathcal{H o m}_{A}\left(C^{\Gamma}(A \mid \mathbb{k})_{*}, M\right) \subseteq \operatorname{Hom}_{A}\left(C^{\Gamma}(A \mid \mathbb{k})_{*}, M\right)
$$


of continuous cochains whose cohomology $\mathscr{H} \Gamma^{*}(A \mid \mathbb{k} ; M)$ we call the continuous $\Gamma$-cohomology of $A$ with coefficients in $M$. Note that continuous cochains can be expressed as the inverse limit

$$
\mathscr{H o m}_{A}\left(C^{\Gamma}(A \mid \mathbb{k})_{*}, M\right)=\lim _{k} \operatorname{Hom}_{A / \mathfrak{m}^{k} A}\left(C^{\Gamma}\left(A / \mathfrak{m}^{k} A \mid \mathbb{k} / \mathfrak{m}^{k}\right)_{*}, M / \mathfrak{m}^{k} M\right)
$$

if $M$ is Hausdorff.

Lemma 2.1. If $(A, \mathbb{k})$ is a topological algebra as above whose completion $\widehat{A}$ is countably free on a topological basis as a $\widehat{\mathbb{k}}$-module and if $M$ is an A-module such that $M / \mathfrak{m}^{k} M$ is Artinian and $M$ is complete, then $\operatorname{Hom}_{A}\left(C^{\Gamma}(A \mid \mathbb{k})_{n}, M\right)$ is linearly compact in each degree $n$.

Proof. As $M$ is complete,

$$
\begin{aligned}
\operatorname{Hom}_{A}\left(C^{\Gamma}(A \mid \mathbb{k})_{n}, M\right) & =\operatorname{Hom}_{A}\left(C^{\Gamma}(A \mid \mathbb{k})_{n}, \lim _{k} M / \mathfrak{m}^{k} M\right) \\
& =\lim _{k} \operatorname{Hom}_{A}\left(C^{\Gamma}(A \mid \mathbb{k})_{n}, M / \mathfrak{m}^{k} M\right) .
\end{aligned}
$$

For any fixed $k$, the homomorphisms from $C^{\Gamma}(A \mid \mathbb{k})_{n}$ to the quotient $M / \mathfrak{m}^{k} M$ factor through

$$
\begin{aligned}
C^{\Gamma}\left(A / \mathfrak{m}^{k} A \mid \mathbb{k} / \mathfrak{m}^{k}\right)_{n} & =\bigoplus_{s+r=n} \operatorname{Lie}(s+1)^{*} \otimes \mathbb{k} / \mathfrak{m}^{k}\left[\Sigma_{s+1}\right]^{\otimes r} \otimes\left(A / \mathfrak{m}^{k} A\right)^{\otimes(s+2)} \\
& =C^{\Gamma}\left(\widehat{A} / \mathfrak{m}^{k} \widehat{A} \mid \widehat{\mathbb{k}} / \mathfrak{m}^{k}\right)_{n}
\end{aligned}
$$

As we assumed that $\widehat{A}$ is countably free on a topological basis, the quotient $\widehat{A} / \mathrm{m}^{k} \widehat{A}$ is free on a countable basis. Hence for any homomorphism $f$ in $\operatorname{Hom}_{A}\left(C^{\Gamma}(A \mid \mathbb{k})_{n}, M\right)$ there is a $j$ such that $f$ factors over the finitely-generated free submodule $C(j)_{n} \subseteq$ $C^{\Gamma}\left(\widehat{A} / \mathfrak{m}^{k} \widehat{A} \mid \widehat{k} / \mathfrak{m}^{k}\right)_{n}$ spanned by the first $j$ generators of $C^{\Gamma}\left(\widehat{A} / \mathfrak{m}^{k} \widehat{A} \mid \widehat{k} / \mathfrak{m}^{k}\right)_{n}$. Therefore

$$
\begin{aligned}
\operatorname{Hom}_{A}\left(C^{\Gamma}(A \mid \mathbb{k})_{n}, M\right) & =\lim _{k} \operatorname{Hom}_{A}\left(C^{\Gamma}(A \mid \mathbb{k})_{n}, M / \mathfrak{m}^{k} M\right) \\
& =\lim _{k} \operatorname{Hom}_{\widehat{A}}\left(C^{\Gamma}\left(\widehat{A} / \mathfrak{m}^{k} \widehat{A} \mid \widehat{\mathbb{k}} / \mathfrak{m}^{k}\right)_{n}, M / \mathfrak{m}^{k} M\right) \\
& =\lim _{k} \lim _{j} \operatorname{Hom}_{\widehat{A}}\left(C(j)_{n}, M / \mathfrak{m}^{k} M\right) \\
& =\lim _{k} \lim _{j} \prod_{i=1}^{j} M / \mathfrak{m}^{k} M .
\end{aligned}
$$

Therefore $\operatorname{Hom}_{A}\left(C^{\Gamma}(A \mid \mathbb{k})_{n}, M\right)$ is a limit of Artinian modules and thus linearly compact. 
Notice that the above inclusion of complexes induces a forgetful homomorphism

$$
\rho: \mathcal{H} \Gamma^{*}(A \mid \mathbb{k} ; M) \longrightarrow \mathrm{H} \Gamma^{*}(A \mid \mathbb{k} ; M) .
$$

Recall that if $M$ is $\mathfrak{m}$-adically complete and Hausdorff, there is a short exact sequence

$$
0 \rightarrow M \longrightarrow \prod_{k} M / \mathfrak{m}^{k} M \stackrel{\mathrm{id}-\sigma}{\longrightarrow} \prod_{k} M / \mathfrak{m}^{k} M \rightarrow 0,
$$

where $\sigma$ is the shift-reduction map. From (2.1) and (2.3) we deduce a Milnor exact sequence relating $\mathscr{H} \Gamma^{*}$ to ordinary $\Gamma$-cohomology for complete coefficient modules. For a similar result see [7].

Proposition 2.2. As above, let $\mathbb{k}$ be Noetherian with maximal ideal $\mathfrak{m}$. Let $M$ be a complete Hausdorff topological module over $\widehat{A}$ which is finitely-generated over $\widehat{\mathbb{k}}$. Then for each $n$ there is a short exact sequence

$$
\begin{aligned}
0 \rightarrow \lim _{k}{ }^{1} \mathrm{H} \Gamma^{n-1}\left(A / \mathfrak{m}^{k} A \mid \mathbb{k} / \mathfrak{m}^{k} ;\right. & \left.M / \mathfrak{m}^{k} M\right) \longrightarrow \mathscr{H} \Gamma^{n}(\widehat{A} \mid \widehat{\mathbb{k}} ; M) \\
& \longrightarrow \lim _{k} H \Gamma^{n}\left(A / \mathfrak{m}^{k} A \mid \mathbb{k} / \mathfrak{m}^{k} ; M / \mathfrak{m}^{k} M\right) \rightarrow 0 .
\end{aligned}
$$

This leads to some useful calculational results, versions of which have previously appeared in [28], [29]. Notice that for any $\widehat{A}$-module $M$ and $k \geqslant 1$, there is a natural reduction homomorphism

$$
\mathrm{H} \Gamma^{n}(\widehat{A} \mid \widehat{\mathbb{k}} ; M) \longrightarrow \mathrm{H} \Gamma^{n}(A \mid \mathbb{k} ; M) \longrightarrow \mathrm{H} \Gamma^{n}\left(A / \mathfrak{m}^{k} A \mid \mathbb{k} / \mathfrak{m}^{k} ; M / \mathfrak{m}^{k} M\right),
$$

compatible with respect to different values of $k$. In turn there is a homomorphism

$$
\mathrm{H} \Gamma^{n}(\widehat{A} \mid \widehat{\mathbb{k}} ; M) \longrightarrow \mathrm{H} \Gamma^{n}(A \mid \mathbb{k} ; M) \longrightarrow \lim _{k} \mathrm{H} \Gamma^{n}\left(A / \mathfrak{m}^{k} A \mid \mathbb{k} / \mathfrak{m}^{k} ; M / \mathfrak{m}^{k} M\right)
$$

The following result was inspired by [28, lemma 15.6].

Corollary 2.3. Let $M$ be an $\widehat{A}$-module which is complete and Hausdorff with respect to the $\mathfrak{m}$-adic topology and finitely-generated as a $\widehat{\mathbb{k}}$-module. Let $\widehat{A}$ be countably free on a topological basis. Then the natural homomorphism $\rho$ induces an isomorphism

$$
\mathscr{H} \Gamma^{*}(\widehat{A} \mid \widehat{\mathbb{k}} ; M) \cong \mathrm{H} \Gamma^{*}(\widehat{A} \mid \widehat{\mathbb{k}} ; M) \text {. }
$$

In addition

$$
\lim _{k}^{1} \mathrm{H} \Gamma^{n-1}\left(A / \mathfrak{m}^{k} A \mid \mathbb{k} / \mathfrak{m}^{k} ; M / \mathfrak{m}^{k} M\right)=0
$$

and the natural homomorphisms induce isomorphisms

$$
\begin{aligned}
\mathrm{H} \Gamma^{*}(A \mid \mathbb{k} ; M) & \cong \mathrm{H} \Gamma^{*}(\widehat{A} \mid \widehat{\mathbb{k}} ; M) \\
& \cong \mathscr{H} \Gamma^{*}(\widehat{A} \mid \widehat{\mathbb{k}} ; M) \\
& \cong \lim _{k} \mathrm{H} \Gamma^{n}\left(A / \mathfrak{m}^{k} A \mid \mathbb{k} / \mathfrak{m}^{k} ; M / \mathfrak{m}^{k} M\right) .
\end{aligned}
$$


Proof. Using the naturality provided by (2.4) we obtain a diagram of short exact sequences from the Milnor exact sequence of Proposition 2.2 into the one for $\mathrm{H} \Gamma^{n}(\widehat{A} \mid \widehat{\mathbb{k}} ; M)$. As the homomorphisms at either end are identities, the natural map $\mathscr{H} \Gamma^{*}(\widehat{A} \mid \widehat{\mathbb{k}} ; M) \longrightarrow \mathrm{H} \Gamma^{*}(\widehat{A} \mid \widehat{\mathbb{k}} ; M)$ is an isomorphism.

Under the assumptions, the cochain complex for $\Gamma$-cohomology is linearly compact in each degree, by Lemma 2.1. Hence by Proposition 1.4, $\Gamma$-cohomology is also linearly compact in each degree. Therefore

$$
\lim _{k}^{1} \mathrm{H} \Gamma^{n-1}\left(A / \mathfrak{m}^{k} \mid \mathbb{k} / \mathfrak{m}^{k} ; M / \mathfrak{m}^{k} M\right)=0 .
$$

So in this case

$$
\mathscr{H} \Gamma^{*}(\widehat{A} \mid \widehat{\mathbb{k}} ; M) \cong \mathrm{H} \Gamma^{*}(\widehat{A} \mid \widehat{\mathbb{k}} ; M) \cong \lim _{k} \mathrm{H} \Gamma^{n}\left(A / \mathfrak{m}^{k} A \mid \mathbb{k} / \mathfrak{m}^{k} ; M / \mathfrak{m}^{k} M\right) .
$$

Remark 2.4. Analogous ideas apply to Hochschild cohomology for which a continuous version appears in [7].

The following result which will be used in Section 5 .

Proposition 2.5. Let $\mathbb{k}$ be Noetherian with maximal ideal $\mathfrak{m}$ and let $\widehat{\mathbb{k}}=\lim _{k} \mathbb{k} / \mathfrak{m}^{k}$. For any $\mathbb{k}$-algebra $A$ with the $\mathfrak{m}$-adic topology for which $\widehat{A}$ is topologically free on a countable basis there is a long exact sequence

$$
\begin{aligned}
\cdots \longrightarrow \mathrm{H} \Gamma^{n-1}(A \mid \mathbb{k} ; \widehat{\mathbb{k}} / \mathbb{k}) & \longrightarrow \mathrm{H} \Gamma^{n}(A \mid \mathbb{k} ; \mathbb{k}) \longrightarrow \mathrm{H} \Gamma^{n}(A \mid \mathbb{k} ; \widehat{\mathbb{k}}) \\
& \longrightarrow \mathrm{H} \Gamma^{n}(A \mid \mathbb{k} ; \widehat{\mathbb{k}} / \mathbb{k}) \longrightarrow \mathrm{H} \Gamma^{n+1}(A \mid \mathbb{k} ; \mathbb{k}) \longrightarrow \cdots
\end{aligned}
$$

Making use of the isomorphism $\mathrm{H} \Gamma^{n}(\widehat{A} \mid \widehat{\mathbb{k}} ; \widehat{\mathbb{k}}) \cong \mathrm{H} \Gamma^{n}(A \mid \mathbb{k} ; \widehat{\mathbb{k}})$, we also obtain an analogous exact sequence for $\mathrm{H} \Gamma^{n}(\widehat{A} \mid \widehat{k} ; \widehat{\mathbb{k}})$.

Proof. The short exact sequence

$$
0 \rightarrow \mathbb{k} \longrightarrow \widehat{\mathbb{k}} \longrightarrow \widehat{\mathbb{k}} / \mathbb{k} \rightarrow 0
$$

of coefficients together with the last isomorphism from Proposition 2.3 yields this long exact sequence.

Finally, we record a result on the $\Gamma$-(co)homology of formally étale algebras that we will repeatedly use. We call an algebra formally étale if it is a colimit of étale algebras.

Lemma 2.6. If $(A, \mathbb{k})$ is a formally étale algebra then for any A-module $M$,

$$
\mathrm{H} \Gamma_{*}(A \mid \mathbb{k} ; M)=0=\mathrm{H} \Gamma^{*}(A \mid \mathbb{k} ; M) .
$$


Proof. By [33, theorem 6.8 (3)], $\Gamma$-homology and cohomology vanishes for étale algebras. Also, $\Gamma$-homology commutes with colimits. Hence if $A=\operatorname{colim}_{r} A_{r}$ with $A_{r}$ étale, for any $A$-module $M$ we have

$$
\mathrm{H} \Gamma_{*}(A \mid \mathbb{k} ; M)=\underset{r}{\operatorname{colim}} \mathrm{H} \Gamma_{*}\left(A_{r} \mid \mathbb{k} ; M\right)=0 .
$$

The universal coefficient spectral sequence

$$
\mathrm{E}_{2}^{*, *}=\operatorname{Ext}_{A}^{* * *}\left(\mathrm{H} \Gamma_{*}(A \mid \mathbb{k} ; A), M\right) \Longrightarrow \mathrm{H} \Gamma^{*}(A \mid \mathbb{k} ; M)
$$

has trivial $\mathrm{E}_{2}$-term, therefore $\mathrm{H} \Gamma^{*}(A \mid \mathbb{k} ; M)=0$.

\section{Rings of numerical polynomials}

We need to describe some properties of rings of numerical polynomials which appeared in a topological setting in [3], [12] and we follow these sources in our discussion. As topological motivation, we remark that A can be identified with $K U_{0} \mathbb{C P} \infty$ and $\mathrm{A}^{\text {st }}$ with $K U_{0} K U$ and we will calculate the $\Gamma$-cohomology of $K U_{0} K U$ later. By definition,

$$
\begin{aligned}
\mathrm{A} & =\{f(w) \in \mathbb{Q}[w]: \text { for all } n \in \mathbb{Z}, f(n) \in \mathbb{Z}\}, \\
\mathrm{A}^{\mathrm{st}} & =\left\{f(w) \in \mathbb{Q}\left[w, w^{-1}\right]: \text { for all } n \in \mathbb{Z}-\{0\}, f(n) \in \mathbb{Z}[1 / n]\right\}
\end{aligned}
$$

are the rings of numerical and stably numerical polynomials (over $\mathbb{Z}$ ). If $x, y$ are indeterminates, we can work in any of the rings $\mathrm{A}[x, y], \mathrm{A}^{\mathrm{st}}[x, y]$ or $\mathbb{Q}\left[w, w^{-1}\right][x, y]$.

We will make use of the binomial coefficient functions

$$
c_{n}(w)=\left(\begin{array}{l}
w \\
n
\end{array}\right)=\frac{w(w-1) \ldots(w-n+1)}{n !} \in A \subseteq \mathbb{Q}[w]
$$

which can be encoded in the generating function

$$
(1+x)^{w}=\sum_{n \geqslant 0} c_{n}(w) x^{n} \in \mathrm{A}[x] \subseteq \mathbb{Q}[w][x] .
$$

Notice that this satisfies the formal identity

$$
(1+x)^{w}(1+y)^{w}=(1+(x+y+x y))^{w} .
$$

Thus we have

$$
c_{m}(w) c_{n}(w)=\left(\begin{array}{c}
m+n \\
m
\end{array}\right) c_{m+n}(w)+(\text { terms of lower degree }) \text { for } m, n \geqslant 0 .
$$


Theorem 3.1 ([2], [12]).

(a) A is a free $\mathbb{Z}$-module with a basis consisting of the $c_{n}(w)$ for $n \geqslant 0$.

(b) $\mathrm{A}^{\text {st }}$ is the localization $\mathrm{A}^{\mathrm{st}}=\mathrm{A}\left[w^{-1}\right]$ and it is a free $\mathbb{Z}$-module on a countable basis.

Describing explicit $\mathbb{Z}$-bases for $\mathrm{A}^{\text {st }}$ is a non-trivial task, see [16], [23]. On the other hand, the multiplicative structure of the $\mathbb{Z}$-algebra $\mathrm{A}^{\text {st }}$ is in some ways more understandable. Our next result describes some generators for $\mathrm{A}^{\text {st }}$.

Theorem 3.2 ([3], [12]).

(a) The $\mathbb{Z}$-algebra $\mathrm{A}$ is generated by the elements $c_{m}(w)$ with $m \geqslant 1$ subject to the relations of (3.2).

(b) The $\mathbb{Z}$-algebra, $\mathrm{A}^{\text {st }}$ is generated by the elements $w^{-1}$ and $c_{m}(w)$ with $m \geqslant 1$.

(c) We have

$$
\mathrm{A} \otimes \mathbb{Q}=\mathbb{Q}[w], \quad \mathrm{A}^{\mathrm{st}} \otimes \mathbb{Q}=\mathbb{Q}\left[w, w^{-1}\right] .
$$

For the localizations of the rings $\mathrm{A}$ and $\mathrm{A}^{\mathrm{st}}$ at any prime $p$ we have

$$
\begin{aligned}
& \mathrm{A}_{(p)}=\left\{f(w) \in \mathbb{Q}[w]: \text { for all } u \in \mathbb{Z}_{(p)}, f(u) \in \mathbb{Z}_{(p)}\right\}, \\
& \mathrm{A}_{(p)}^{\mathrm{st}}=\left\{f(w) \in \mathbb{Q}[w]: \text { for all } u \in \mathbb{Z}_{(p)}^{\times}, f(u) \in \mathbb{Z}_{(p)}\right\} .
\end{aligned}
$$

Theorem 3.3 ([2], [4, proposition 2.5], [12]).

(a) $\mathrm{A}_{(p)}$ is a free $\mathbb{Z}_{(p)}$-module with a basis consisting of the monomials in the binomial coefficient functions

$$
w^{r_{0}} c_{p}(w)^{r_{1}} c_{p^{2}}(w)^{r_{2}} \ldots c_{p^{\ell}}(w)^{r_{\ell}},
$$

where $r_{k}=0,1, \ldots, p-1$.

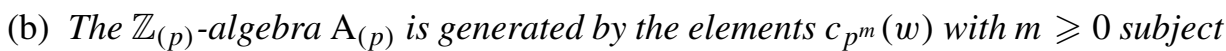
to relations of the form

$$
c_{p^{m}}(w)^{p}-c_{p^{m}}(w)=p d_{m+1}(w),
$$

where $d_{m+1}(w) \in \mathrm{A}_{(p)}$ has $\operatorname{deg} d_{m+1}(w)=p^{m+1}$. In fact the monomials

$$
w^{r_{0}} d_{1}(w)^{r_{1}} d_{2}(w)^{r_{2}} \ldots d_{\ell}(w)^{r_{\ell}},
$$

where $r_{k}=0,1, \ldots, p-1$, form a basis of $\mathrm{A}_{(p)}$ over $\mathbb{Z}_{(p)}$ and are subject to multiplicative relations of the form

$$
d_{m}(w)^{p}-d_{m}(w)=p d_{m+1}^{\prime}(w),
$$

where $\operatorname{deg} d_{m+1}^{\prime}(w)=p^{m+1}$. 
(c) $\mathrm{A}_{(p)}^{\mathrm{st}}$ is the localization $\mathrm{A}_{(p)}^{\mathrm{st}}=\mathrm{A}_{(p)}\left[w^{-1}\right]$ and it is a free $\mathbb{Z}_{(p)}$-module on a countable basis.

(d) The $\mathbb{Z}_{(p)}$-algebra, $\mathrm{A}_{(p)}^{\mathrm{st}}$ is generated by the elements $w$ and $e_{m}(w) \in \mathrm{A}_{(p)}^{\mathrm{st}}$ for $m \geqslant 1$ defined recursively by

$$
w^{p-1}-1=p e_{1}(w), \quad e_{m}(w)^{p}-e_{m}(w)=p e_{m+1}(w) \text { for } m \geqslant 1 .
$$

Corollary 3.4. Let $p$ be a prime.

(a) As $\mathbb{F}_{p}$-algebras,

$$
\begin{aligned}
\mathrm{A} / p \mathrm{~A} & =\mathbb{F}_{p}\left[c_{p^{m}}(w): m \geqslant 0\right] /\left(c_{p^{m}}(w)^{p}-c_{p^{m}}(w): m \geqslant 0\right), \\
\mathrm{A}^{\mathrm{st}} / p \mathrm{~A}^{\mathrm{st}} & =\mathbb{F}_{p}\left[w, e_{m}(w): m \geqslant 0\right] /\left(w^{p-1}-1, e_{m}(w)^{p}-e_{m}(w): m \geqslant 1\right) .
\end{aligned}
$$

Hence these algebras are formally étale over $\mathbb{F}_{p}$.

(b) For $n \geqslant 1, \mathrm{~A} / p^{n} \mathrm{~A}$ and $\mathrm{A}^{\mathrm{st}} / p^{n} \mathrm{~A}^{\mathrm{st}}$ are formally étale over $\mathbb{Z} / p^{n}$.

(c) The p-adic completions $\mathrm{A}_{p}=\lim _{n} \mathrm{~A} / p^{n} \mathrm{~A}$ and $\mathrm{A}_{p}^{\mathrm{st}}=\lim _{n} \mathrm{~A}^{\mathrm{st}} / p^{n} \mathrm{~A}^{\mathrm{st}}$ are formally étale over $\mathbb{Z}_{p}$.

(d) $\mathrm{A}_{p}$ and $\mathrm{A}_{p}^{\text {st }}$ are free topological $\mathbb{Z}_{p}$-modules on countable bases. Therefore they are both compact and Hausdorff.

Proof. Parts (b) and hence (c) can be proved by induction on $n \geqslant 1$ using the infinitedimensional Hensel lemma of [7, 3.9]. The case $n=1$ is immediate from (a). Suppose that we have found a sequence of elements $s_{0}, s_{1}, \ldots, s_{k}, \ldots \in \mathrm{A}_{(p)}$ satisfying

$$
s_{m}^{p}-s_{m} \equiv 0\left(\bmod p^{n}\right) \quad \text { for } m \geqslant 0 .
$$

Taking $s_{m}^{\prime}=s_{m}+\left(s_{m}^{p}-s_{m}\right)$ we find that

$$
\begin{aligned}
s_{m}^{\prime}{ }^{p}-s_{m}^{\prime} & =\left(s_{m}+\left(s_{m}^{p}-s_{m}\right)\right)^{p}-\left(s_{m}+\left(s_{m}^{p}-s_{m}\right)\right) \\
& \equiv s_{m}^{p}-\left(s_{m}+\left(s_{m}^{p}-s_{m}\right)\right)\left(\bmod p^{n+1}\right) \\
& =0 .
\end{aligned}
$$

Hence for every $n$ we can inductively produce such elements $s_{n, m} \in \mathrm{A}_{(p)}$ for which

$$
\begin{aligned}
\mathrm{A} / p^{n} \mathrm{~A} & =\mathbb{Z} / p^{n}\left[s_{n, m}: m \geqslant 0\right] /\left(s_{n, m}^{p}-s_{n, m}: m \geqslant 0\right) \\
& =\bigotimes_{m \geqslant 0} \mathbb{Z} / p^{n}\left[s_{n, m}\right] /\left(s_{n, m}^{p}-s_{n, m}\right) .
\end{aligned}
$$

Now passing to $p$-adic limits we obtain elements

$$
s_{m}=\lim _{n \rightarrow \infty} s_{n, m} \in \mathrm{A}_{p}
$$


for which

$$
s_{m}^{p}-s_{m}=0 .
$$

In these cases we obtain for the module of Kähler differentials

$$
\Omega_{\left(\mathrm{A} / p^{n} \mathrm{~A}\right) / \mathbb{Z} / p^{n}}^{1}=0=\Omega_{\mathrm{A}_{p} / \mathbb{Z}_{p}}^{1}
$$

Part (d) is related to Mahler's Theorem and a suitable exposition of this can be found in [4].

There are two natural choices of augmentation for A, namely evaluation at 0 or 1 ,

$$
\begin{aligned}
& \varepsilon_{+}: \mathrm{A} \longrightarrow \mathbb{Z} ; \quad \varepsilon_{+} f(w)=f(0), \\
& \varepsilon_{\times}: \mathrm{A} \longrightarrow \mathbb{Z} ; \quad \varepsilon_{\times} f(w)=f(1) .
\end{aligned}
$$

For our purposes, the latter augmentation will be used. Notice that there is a ring automorphism

$$
\varphi: \mathrm{A} \longrightarrow \mathrm{A} ; \quad \varphi f(w)=f(w+1)
$$

for which $\varepsilon_{+} \varphi=\varepsilon_{\times}$, so these augmentations are not too dissimilar.

\section{The ring of $\mathbb{Z} /(p-1)$-invariants in $A_{(p)}^{\text {st }}$}

In this section, $p$ always denotes an odd prime. The case of $p=2$ is related to $K O$ and the work of Section 7.

Since polynomial functions $\mathbb{Z}_{(p)}^{\times} \longrightarrow \mathbb{Q}$ are continuous with respect to the $p$-adic topology they extend to continuous functions $\mathbb{Z}_{p}^{\times} \longrightarrow \mathbb{Q}_{p}$; such functions which also map $\mathbb{Z}_{(p)}^{\times}$into $\mathbb{Z}_{(p)}$ give continuous functions $\mathbb{Z}_{p}^{\times} \longrightarrow \mathbb{Z}_{p}$. Hence we can regard $\mathrm{A}_{(p)}^{\text {st }}$ as a subring of $\mathbb{Q}_{p}\left[w, w^{-1}\right]$ which in turn can be viewed as a space of continuous functions on the $p$-adic units $\mathbb{Z}_{p}^{\times}$. For $p \geqslant 3$ there is a splitting of topological groups

$$
\mathbb{Z}_{p}^{\times} \cong \mathbb{Z} /(p-1) \times\left(1+p \mathbb{Z}_{p}\right)
$$

where $\mathbb{Z} /(p-1)$ identifies with a subgroup generated by a primitive $(p-1)$-st root of unity $\zeta$. There is also a bicontinuous isomorphism $1+p \mathbb{Z}_{p} \cong \mathbb{Z}_{p}$. by

For an odd prime $p$, the group $\langle\zeta\rangle \cong \mathbb{Z} /(p-1)$ acts continuously on $\mathbb{Q}_{p}\left[w, w^{-1}\right]$

$$
\zeta \cdot f(w)=f(\zeta w)
$$

and it is immediate that this action sends elements of $\mathrm{A}_{(p)}^{\mathrm{st}}$ to continuous functions $\mathbb{Z}_{p}^{\times} \longrightarrow \mathbb{Z}_{p}$. It then makes sense to ask for the subring of $A_{(p)}^{\text {st }}$ fixed by this action, ${ }^{\zeta} \mathrm{A}_{(p)}^{\mathrm{st}}$. We will relate this subring to the algebra of cooperations of the Adams summand in Proposition 6.1.

Recall the elements $e_{m}(w)$ of Theorem 3.3(d). We will write $\bar{e}_{m}(w)$ for $w^{-1} e_{m}(w)$. 
Vol. 80 (2005) $\quad \Gamma$-cohomology of rings of numerical polynomials and $E_{\infty}$ structures 705

Proposition 4.1. As a $\mathbb{Z}_{(p)}$-algebra, ${ }^{\zeta} \mathrm{A}_{(p)}^{\mathrm{st}}$ is generated by the elements $w^{p-1}$ and $\bar{e}_{m}(w)$ for $m \geqslant 1$.

Proof. It is clear that

$$
{ }^{\zeta} \mathbb{Q}\left[w, w^{-1}\right]=\mathbb{Q}\left[w^{p-1}, w^{-(p-1)}\right]
$$

Also, by construction of the $e_{m}(w)$,

$$
\bar{e}_{m}(w) \in{ }^{\zeta} \mathrm{A}_{(p)}^{\mathrm{st}} \subseteq \mathbb{Q}\left[w^{p-1}, w^{-(p-1)}\right] .
$$

Consider the multiplicative idempotent

$$
E_{\zeta}: \mathbb{Q}\left[w, w^{-1}\right] \longrightarrow \mathbb{Q}\left[w, w^{-1}\right] ; \quad E_{\zeta} f(w)=\frac{1}{p-1} \sum_{r=1}^{p-1} f\left(\zeta^{r} w\right) .
$$

Then we have

$$
{ }^{\zeta} \mathrm{A}_{(p)}^{\mathrm{st}}=E_{\zeta} \mathrm{A}_{(p)}^{\mathrm{st}} .
$$

Each element $f(w) \in \mathbb{Q}\left[w, w^{-1}\right]$ has the form

$$
f(w)=f_{0}\left(w^{p-1}\right)+w f_{1}\left(w^{p-1}\right)+\cdots+w^{p-2} f_{p-2}\left(w^{p-1}\right),
$$

where $f_{k}(x) \in \mathbb{Q}\left[x, x^{-1}\right]$, hence

$$
E_{\zeta} f(w)=f_{0}\left(w^{p-1}\right)
$$

From this it follows that ${ }^{\zeta} \mathrm{A}_{(p)}^{\text {st }}$ is generated as a $\mathbb{Z}_{(p)}$-algebra by the stated elements.

\section{Corollary 4.2. The following hold.}

(a) As $\mathbb{F}_{p}$-algebras,

$$
{ }^{\zeta} \mathrm{A}_{(p)}^{\mathrm{st}} / p\left({ }^{\zeta} \mathrm{A}_{(p)}^{\mathrm{st}}\right)=\mathbb{F}_{p}\left[w, \bar{e}_{m}(w): m \geqslant 1\right] /\left(w^{p-1}-1, \bar{e}_{m}(w)^{p}-\bar{e}_{m}(w): m \geqslant 1\right) .
$$

Hence this algebra is formally étale over $\mathbb{F}_{p}$.

(b) For $n \geqslant 1,{ }^{\zeta} \mathrm{A}_{(p)}^{\mathrm{st}} / p^{n}\left({ }^{\zeta} \mathrm{A}_{(p)}^{\mathrm{st}}\right)$ is formally étale over $\mathbb{Z} / p^{n}$.

(c) The p-adic completion ${ }^{\zeta} \mathrm{A}_{p}^{\mathrm{st}}=\lim _{n}{ }^{\zeta} \mathrm{A}_{(p)}^{\mathrm{st}} / p^{n}\left({ }^{\zeta} \mathrm{A}^{\mathrm{st}}\right)_{(p)}$ is formallyétale over $\mathbb{Z}_{p}$. 


\section{The $\Gamma$-cohomology of numerical polynomials}

Recall that $\widehat{\mathbb{Z}} / \mathbb{Z}$ and $\mathbb{Z}_{p} / \mathbb{Z}_{(p)}$ for any prime $p$ are torsion-free divisible groups, so they are both $\mathbb{Q}$-vector spaces which have the same cardinality and (uncountable) dimensions; thus they are isomorphic. Similarly, we have $\widehat{\mathbb{Z}} / \mathbb{Z} \cong \widehat{\mathbb{Q}} / \mathbb{Q}$ and $\mathbb{Z}_{p} / \mathbb{Z}_{(p)} \cong \mathbb{Q}_{p} / \mathbb{Q}$.

In the following, we will always use the augmentations $\varepsilon_{\times}: A \longrightarrow \mathbb{Z}$ and $\varepsilon_{\times}: A^{\text {st }} \longrightarrow \mathbb{Z}$ and their analogues for the $p$-localized versions. In calculating the $\Gamma$-cohomology of A, we would obtain the same result using $\varepsilon_{+}$because of the existence of the automorphism $\varphi$ of (3.4).

Theorem 5.1. Let $R$ be an augmented commutative $\mathbb{Z}$-algebra. Assume that, at each prime $p$, the p-completion $R_{p}$ is topologically free on a countable basis. Suppose that for all primes $p$ and $k \geqslant 1, R / p^{k}$ is a formally étale algebra over $\mathbb{Z} / p^{k}$. Then for all $s \geqslant 0$,

$$
\mathrm{H} \Gamma^{s}(R \mid \mathbb{Z} ; \mathbb{Z}) \cong \mathrm{H} \Gamma^{s-1}\left(R_{\mathbb{Q}} \mid \mathbb{Q} ; \mathbb{Q}\right) .
$$

Proof. For each natural number $n$, we may write

$$
n=\prod_{p} p^{\operatorname{ord}_{p} n}
$$

where the product is taken over all primes $p$. The Chinese Remainder Theorem provides splittings

$$
\begin{aligned}
\mathbb{Z} / n & =\prod_{p} \mathbb{Z} / p^{\operatorname{ord}_{p} n}, \\
\widehat{\mathbb{Z}} & =\prod_{p} \mathbb{Z}_{p} .
\end{aligned}
$$

Applying the Transitivity Sequence and using that $R_{(p)}$ is étale over $R$, we obtain that at each prime $p$

$$
\mathrm{H} \Gamma^{*}\left(R_{(p)} \mid \mathbb{Z}(p) ; \widehat{\mathbb{Z}}\right) \cong \mathrm{H} \Gamma^{*}(R \mid \mathbb{Z} ; \widehat{\mathbb{Z}}) .
$$

Therefore by Corollary 2.3 we have

$$
\mathrm{H} \Gamma^{*}(R \mid \mathbb{Z} ; \widehat{\mathbb{Z}})=\prod_{p} \mathrm{H} \Gamma^{*}\left(R \mid \mathbb{Z} ; \mathbb{Z}_{p}\right) \cong \prod_{p} \mathrm{H} \Gamma^{*}\left(R_{p} \mid \mathbb{Z}_{p} ; \mathbb{Z}_{p}\right) .
$$

For the second isomorphism, using Corollary 2.3 and the linear compactness of $\Gamma$-cohomology provided by Proposition 1.4 , we can express $\mathrm{H} \Gamma^{*}\left(R \mid \mathbb{Z} ; \mathbb{Z}_{p}\right)$ as the inverse limit of the groups $\mathrm{H} \Gamma^{*}\left(R \mid \mathbb{Z} ; \mathbb{Z} / p^{n}\right)$. Here the coefficients $\mathbb{Z} / p^{n}$ eliminate the effect of all the $p$-divisible elements, therefore $\mathrm{H} \Gamma^{*}\left(R \mid \mathbb{Z} ; \mathbb{Z} / p^{n}\right)$ reduces to $\mathrm{H} \Gamma^{*}\left(R_{p} \mid \mathbb{Z}_{p} ; \mathbb{Z} / p^{n}\right)$, where $R_{p}$ denotes the $p$-adic completion of $R$. 
Now for each $k \geqslant 1$, the assumption that $R / p^{k}$ is formally étale over $\mathbb{Z} / p^{k}$ ensures that

$$
\mathrm{H} \Gamma^{*}\left(R / p^{k} \mid \mathbb{Z} / p^{k} ; \mathbb{Z} / p^{k}\right)=0 .
$$

Therefore we obtain

$$
\begin{aligned}
\mathrm{H} \Gamma^{*}\left(R_{p} \mid \mathbb{Z}_{p} ; \mathbb{Z}_{p}\right) & =\lim _{k} \mathrm{H} \Gamma^{*}\left(R / p^{k} \mid \mathbb{Z} / p^{k} ; \mathbb{Z} / p^{k}\right)=0 \\
& =\lim _{k}^{1} \mathrm{H} \Gamma^{*}\left(R / p^{k} \mid \mathbb{Z} / p^{k} ; \mathbb{Z} / p^{k}\right) .
\end{aligned}
$$

For each $n$, Proposition 2.5 implies that

$$
\begin{aligned}
\mathrm{H} \Gamma^{n}\left(R_{(p)} \mid \mathbb{Z}_{(p)} ; \mathbb{Z}_{(p)}\right) & =\mathrm{H} \Gamma^{n-1}\left(R_{(p)} \mid \mathbb{Z}_{(p)} ; \mathbb{Z}_{p} / \mathbb{Z}_{(p)}\right), \\
\mathrm{H} \Gamma^{n}(R \mid \mathbb{Z} ; \mathbb{Z}) & =\mathrm{H} \Gamma^{n-1}(R \mid \mathbb{Z} ; \widehat{\mathbb{Z}} / \mathbb{Z}) .
\end{aligned}
$$

As $\mathbb{Z}_{p} / \mathbb{Z}_{(p)}$ and $\widehat{\mathbb{Z}} / \mathbb{Z}$ are $\mathbb{Q}$-vector spaces, for all $n \neq 0$ we obtain

$$
\mathrm{H} \Gamma^{n}\left(R_{(p)} \mid \mathbb{Z}_{(p)} ; \mathbb{Z}_{p} / \mathbb{Z}_{(p)}\right) \cong \mathrm{H} \Gamma^{n}\left(R_{\mathbb{Q}} \mid \mathbb{Q} ; \mathbb{Z}_{p} / \mathbb{Z}_{(p)}\right)
$$

and similarly

$$
\mathrm{H} \Gamma^{n}(R \mid \mathbb{Z} ; \widehat{\mathbb{Z}} / \mathbb{Z}) \cong \mathrm{H} \Gamma^{n}\left(R_{\mathbb{Q}} \mid \mathbb{Q} ; \widehat{\mathbb{Z}} / \mathbb{Z}\right)
$$

Corollary 5.2. We have

$$
\mathrm{H} \Gamma^{n}\left(\mathrm{~A}^{\mathrm{st}} \mid \mathbb{Z} ; \mathbb{Z}\right)=\mathrm{H} \Gamma^{n}(\mathrm{~A} \mid \mathbb{Z} ; \mathbb{Z})= \begin{cases}\widehat{\mathbb{Z}} / \mathbb{Z} & \text { if } n=1, \\ 0 & \text { otherwise. }\end{cases}
$$

For each prime $p$,

$$
\mathrm{H} \Gamma^{n}\left(\mathrm{~A}_{(p)}^{\mathrm{st}} \mid \mathbb{Z}_{(p)} ; \mathbb{Z}_{(p)}\right)=\mathrm{H} \Gamma^{n}\left(\mathrm{~A}_{(p)} \mid \mathbb{Z}_{(p)} ; \mathbb{Z}_{(p)}\right)= \begin{cases}\mathbb{Z}_{p} / \mathbb{Z}_{(p)} & \text { if } n=1, \\ 0 & \text { otherwise }\end{cases}
$$

Proof. Since $\mathrm{A}\left[w^{-1}\right]$ is étale over $\mathrm{A}$, both of the $\Gamma$-cohomology groups

$$
\mathrm{H} \Gamma^{*}\left(\mathrm{~A}\left[w^{-1}\right] \mid \mathrm{A} ; \mathbb{Z}\right) \text { and } \quad \mathrm{H}^{*}\left(\mathrm{~A}_{(p)}\left[w^{-1}\right] \mid \mathrm{A}_{(p)} ; \mathbb{Z}\right)
$$

vanish. The Transitivity Theorem [33, 3.4] implies that there are isomorphisms

$$
\begin{aligned}
& \mathrm{H} \Gamma^{*}(\mathrm{~A} \mid \mathbb{Z} ; \mathbb{Z}) \cong \mathrm{H} \Gamma^{*}\left(\mathrm{~A}\left[w^{-1}\right] \mid \mathbb{Z} ; \mathbb{Z}\right)=\mathrm{H} \Gamma^{*}\left(\mathrm{~A}^{\mathrm{st}} \mid \mathbb{Z} ; \mathbb{Z}\right), \\
& \mathrm{H} \Gamma^{*}\left(\mathrm{~A}_{(p)} \mid \mathbb{Z}_{(p)} ; \mathbb{Z}_{(p)}\right) \cong \mathrm{H} \Gamma^{*}\left(\mathrm{~A}_{(p)}\left[w^{-1}\right] \mid \mathbb{Z}_{(p)} ; \mathbb{Z}_{(p)}\right)=\mathrm{H} \Gamma^{*}\left(\mathrm{~A}_{(p)}^{\mathrm{st}} \mid \mathbb{Z}_{(p)} ; \mathbb{Z}_{(p)}\right),
\end{aligned}
$$

hence it suffices to prove the result for $\mathrm{A}$ and $\mathrm{A}_{(p)}$. 
Corollary 3.4 ensures that $\mathrm{A} / p^{k} \mathrm{~A}$ and therefore $\mathrm{A}_{(p)} / p^{k} \mathrm{~A}_{(p)}$ as well is formally étale over $\mathbb{Z} / p^{k}$ for all $k \geqslant 1$. Now Corollaries 3.4(d) and 1.3 together guarantee that the cochains for $\Gamma$-cohomology fulfil the linear compactness requirements of Theorem 5.1. Thus we can apply this theorem and obtain the vanishing result for $\Gamma$-cohomology in dimensions different from one.

By $[29$, theorem 4.1$]$ and the fact that $\mathbb{Z}_{p} / \mathbb{Z}_{(p)}$ and $\widehat{\mathbb{Z}} / \mathbb{Z}$ are $\mathbb{Q}$-vector spaces, we have

$$
\begin{aligned}
\mathrm{H} \Gamma^{*}\left(\mathrm{~A}_{(p)} \mid \mathbb{Z}_{(p)} ; \mathbb{Z}_{p} / \mathbb{Z}_{(p)}\right) & =\mathrm{H} \Gamma^{*}\left(\mathrm{~A} \otimes \mathbb{Q} \mid \mathbb{Q} ; \mathbb{Z}_{p} / \mathbb{Z}_{(p)}\right) \\
& =\mathrm{H} \Gamma^{*}\left(\mathbb{Q}[w] \mid \mathbb{Q} ; \mathbb{Z}_{p} / \mathbb{Z}_{(p)}\right)=\mathbb{Z}_{p} / \mathbb{Z}_{(p)}
\end{aligned}
$$

and

$$
\mathrm{H} \Gamma^{*}(\mathrm{~A} \mid \mathbb{Z} ; \widehat{\mathbb{Q}} / \mathbb{Q})=\mathrm{H} \Gamma^{*}(\mathrm{~A} \otimes \mathbb{Q} \mid \mathbb{Q} ; \widehat{\mathbb{Z}} / \mathbb{Z})=\mathrm{H} \Gamma^{*}(\mathbb{Q}[w] \mid \mathbb{Q} ; \widehat{\mathbb{Z}} / \mathbb{Z})=\widehat{\mathbb{Z}} / \mathbb{Z}
$$

Thus we obtain

$$
\mathrm{H} \Gamma^{n}\left(\mathrm{~A}_{(p)} \mid \mathbb{Z}_{(p)} ; \mathbb{Z}_{(p)}\right)= \begin{cases}\mathbb{Z}_{p} / \mathbb{Z}_{(p)} & \text { if } n=1, \\ 0 & \text { otherwise }\end{cases}
$$

and

$$
\mathrm{H} \Gamma^{n}(\mathrm{~A} \mid \mathbb{Z} ; \mathbb{Z})= \begin{cases}\widehat{\mathbb{Z}} / \mathbb{Z} & \text { if } n=1 \\ 0 & \text { otherwise }\end{cases}
$$

as claimed.

Remark 5.3. Notice, that for the calculations of $\Gamma$-cohomology above we used the formal properties of $\Gamma$-cohomology. As André-Quillen cohomology satisfies analoguous properties, we can transfer the above results to obtain the following:

$$
\begin{aligned}
& \mathrm{AQ}_{n}\left(\mathrm{~A}^{\mathrm{st}} \mid \mathbb{Z} ; \mathbb{Z}\right)=\mathrm{AQ}_{n}(\mathrm{~A} \mid \mathbb{Z} ; \mathbb{Z})= \begin{cases}\mathbb{Q} & \text { if } n=0, \\
0 & \text { if } n \neq 0,\end{cases} \\
& \mathrm{AQ}^{n}\left(\mathrm{~A}^{\mathrm{st}} \mid \mathbb{Z} ; \mathbb{Z}\right)=\mathrm{AQ}^{n}(\mathrm{~A} \mid \mathbb{Z} ; \mathbb{Z})= \begin{cases}\widehat{\mathbb{Q}} / \mathbb{Q} & \text { if } n=1, \\
0 & \text { if } n \neq 1 .\end{cases}
\end{aligned}
$$

The results from Section 4 allow us to calculate the $\Gamma$-cohomology of ${ }^{\zeta} \mathrm{A}_{(p)}^{\mathrm{st}}$ over $\mathbb{Z}_{(p)}$ directly as was done above for $\mathrm{A}^{\text {st }}$. Alternatively, we may use the fact that the extension $\mathrm{A}_{(p)}^{\mathrm{st}} / \zeta \mathrm{A}_{(p)}^{\mathrm{st}}$ is étale since it has the form $B / A$, where $B=A[t] /\left(t^{p-1}-v\right)$ for a unit $v \in A$, where $A$ is a $\mathbb{Z}_{(p)}$-algebra. We can now determine the $\Gamma$-cohomology of $\zeta \mathrm{A}_{(p)}^{\text {st }}$ since the Transitivity Theorem of $[33,3.4]$ gives

Proposition 5.4. For an odd prime $p$,

$$
\mathrm{H} \Gamma^{*}\left({ }^{\zeta} \mathrm{A}_{(p)}^{\mathrm{st}} \mid \mathbb{Z}_{(p)} ; \mathbb{Z}_{(p)}\right)=\mathrm{H} \Gamma^{*}\left(\mathrm{~A}_{(p)}^{\mathrm{st}} \mid \mathbb{Z}_{(p)} ; \mathbb{Z}_{(p)}\right) .
$$




\section{Applications to $E_{\infty}$ structures on $K$-theory}

Robinson [31] has developed an obstruction theory for $E_{\infty}$ structures on a homotopy commutative ring spectrum $E$. Provided $E$ satisfies the following form of the Künneth and universal coefficient theorems for $E_{*} E$

$$
E^{*}\left(E^{\wedge n}\right) \cong \operatorname{Hom}_{E_{*}}\left(E_{*} E^{\otimes n}, E_{*}\right),
$$

then the obstructions lie in groups

$$
\mathrm{H} \Gamma^{n, 2-n}\left(E_{*} E \mid E_{*} ; E_{*}\right),
$$

while the extensions are determined by classes in

$$
\mathrm{H} \Gamma^{n, 1-n}\left(E_{*} E \mid E_{*} ; E_{*}\right) .
$$

Here the bigrading $(s, t)$ involves cohomological degree $s$ and internal degree $t$. Moreover, the relevant values of $n$ are for $n \geqslant 3$.

We want to apply this to the cases of complex $K U$-theory and the Adams summand $E(1)$ of $K U_{(p)}$ at a prime $p$. Recall that

$$
K U_{*}=\mathbb{Z}\left[t, t^{-1}\right], \quad K U_{(p)_{*}}=\mathbb{Z}_{(p)}\left[t, t^{-1}\right], \quad E(1)_{*}=\mathbb{Z}_{(p)}\left[u, u^{-1}\right],
$$

where $t \in K U_{2}$ and $u \in E(1)_{2(p-1)}$. Our next result implies that the relevant conditions mentioned above are both satisfied for $K U$ and $E(1)$.

Proposition 6.1. There are isomorphisms of rings (in fact, of Hopf algebras)

$$
\begin{gathered}
K U_{0} K U \cong \mathrm{A}^{\mathrm{st}}, \quad K U_{(p)_{0}} K U_{(p)} \cong \mathrm{A}_{(p)}^{\mathrm{st}}, \\
E(1)_{0} E(1) \cong{ }^{\zeta} \mathrm{A}_{(p)}^{\mathrm{st}} .
\end{gathered}
$$

Hence,

$$
\begin{gathered}
K U_{*} K U \cong K U_{*} \otimes \mathrm{A}^{\mathrm{st}}, \quad K U_{(p)_{*}} K U_{(p)} \cong K U_{(p)_{*}} \otimes \mathrm{A}_{(p)}^{\mathrm{st}}, \\
E(1)_{*} E(1) \cong E(1)_{*} \otimes{ }^{\zeta} \mathrm{A}_{(p)}^{\mathrm{st}} .
\end{gathered}
$$

Proof. The isomorphisms for $K U$ and $K U_{(p)}$ can be found in [12, p. 392].

Consider $E(1)_{*} E(1)$, the algebra of cooperations for $E(1)$. Since $E(1)$ is Landweber exact, we have

$$
\begin{aligned}
E(1)_{*} E(1) & \cong E(1)_{*} \otimes_{B P_{*}} B P_{*} B P \otimes_{B P_{*}} E(1)_{*} \\
& \cong E(1)_{*}\left[t_{1}, t_{2}, \ldots, V_{1}, V_{1}^{-1}\right] / \text { (relations) },
\end{aligned}
$$


where $V_{1}$ denotes the right unit $\eta_{r}$ applied to $v_{1}$ and the variables $t_{i}$ stem from $B P_{*} B P$. We also write $\bar{w}=v_{1}^{-1} V_{1}$. The relation $v_{1}+p t_{1}-V_{1}=0$ in $E(1)_{*} E(1)$ gives rise to

$$
1-v_{1}^{-1} V_{1}=-p v_{1}^{-1} t_{1} \in E(1)_{0} E(1),
$$

hence on setting $\bar{e}_{1}=v_{1}^{-1} t_{1}$ we have

$$
\bar{w}-1=p \bar{e}_{1} .
$$

Now we may inductively define

$$
\bar{e}_{m}=v_{1}^{-p^{m-1}-\cdots-p-1} t_{m} .
$$

The higher relations

$$
v_{1} t_{k}^{p}-v_{1}^{p^{k}} t_{k}+p t_{k+1}=0 \quad(k \geqslant 1)
$$

can be used to prove the desired relations for the $\bar{e}_{m}$. Taking the $p$-th power we have

$$
\bar{e}_{m}^{p}=v_{1}^{-p^{m}-\cdots-p} t_{m}^{p}
$$

Multiplying the relation

$$
v_{1} t_{m}^{p}-v_{1}^{p^{m}} t_{m}=p t_{m+1}
$$

by $v_{1}^{-p^{m}-\cdots-p-1}$, we obtain

$$
\bar{e}_{m}^{p}-v_{1}^{p^{m}-p^{m}-p^{m-1}-\cdots-p-1} t_{m}=p v_{1}^{-p^{m}-\cdots-p-1} t_{m+1}
$$

which is precisely

$$
\bar{e}_{m}^{p}-\bar{e}_{m}=p \bar{e}_{m+1}
$$

Flat base-change leads to isomorphisms

$$
\begin{aligned}
\mathrm{H} \Gamma^{*, *}\left(K U_{*} K U \mid K U_{*} ; K U_{*}\right) & \cong \mathrm{H} \Gamma^{*}\left(\mathrm{~A}^{\mathrm{st}} \mid \mathbb{Z} ; \mathbb{Z}\right) \otimes K U_{*}, \\
\mathrm{H} \Gamma^{*, *}\left(K U_{(p)_{*}} K U_{(p)} \mid K U_{(p)_{*}} ; K U_{(p)_{*}}\right) & \cong \mathrm{H} \Gamma^{*}\left(\mathrm{~A}_{(p)}^{\mathrm{st}} \mid \mathbb{Z}_{(p)} ; \mathbb{Z}_{(p)}\right) \otimes K U_{(p)_{*},} \\
\mathrm{H} \Gamma^{*, *}\left(E(1)_{*} E(1) \mid E(1)_{*} ; E(1)_{*}\right) & \cong \mathrm{H} \Gamma^{*}\left({ }^{\zeta} \mathrm{A}_{(p)}^{\mathrm{st}} \mid \mathbb{Z}_{(p)} ; \mathbb{Z}_{(p)}\right) \otimes E(1)_{*} .
\end{aligned}
$$

With the help of Corollary 5.2 we can therefore deduce the following.

Theorem 6.2. For a prime $p$ and $n \geqslant 2$,

$$
\begin{aligned}
& \mathrm{H} \Gamma^{n, 2-n}\left(K U_{*} K U \mid K U_{*} ; K U_{*}\right)=0 \\
& =\mathrm{H} \Gamma^{n, 1-n}\left(K U_{*} K U \mid K U_{*} ; K U_{*}\right), \\
& \begin{aligned}
\mathrm{H} \Gamma^{n, 2-n}\left(K U_{(p)_{*}} K U_{(p)} \mid K U_{(p)_{*}} ; K U_{\left.(p)_{*}\right)}\right)=0 \\
=\mathrm{H} \Gamma^{n, 1-n}\left(K U_{(p)_{*}} K U_{(p)} \mid K U_{(p)_{*}} ; K U_{\left.(p)_{*}\right)}\right. \\
\mathrm{H} \Gamma^{n, 2-n}\left(E(1)_{*} E(1) \mid E(1)_{*} ; E(1)_{*}\right)=0 \\
=\mathrm{H} \Gamma^{n, 1-n}\left(E(1)_{*} E(1) \mid E(1)_{*} ; E(1)_{*}\right) .
\end{aligned}
\end{aligned}
$$


Hence $K U, K U_{(p)}$, and $E(1)$ each have a unique $E_{\infty}$ structure.

It is a rather old question whether the connective Adams summand, often denoted by $\ell$, is an $E_{\infty}$ spectrum. The $E_{\infty}$ ring spectrum machinery developed in [26] yields the following general result.

Theorem 6.3. For any $E_{\infty}$ ring spectrum $E$, the connective cover $\longrightarrow$ E possesses a model as an $E_{\infty}$ ring spectrum.

Proof. Proceeding as in [26, proposition VII.3.2], we first take the underlying zeroth space $E_{0}$ of the $E_{\infty}$ ring spectrum $E$, then build a prespectrum $T\left(E_{0}\right)$ out of it using a bar construction which consists of suspensions and the monad for the little convex body (partial) operad. Finally we apply the spectrification functor (there called $\Omega^{\infty}$ ) to $T\left(E_{0}\right)$. By [26, proposition VII.3.2], this has the correct homotopy groups and is an $E_{\infty}$ ring spectrum.

Applying this result, we obtain a canonical $E_{\infty}$ model for the connective cover $\ell \longrightarrow E(1)$.

Proposition 6.4. There is at least one $E_{\infty}$ structure on the connective Adams summand $\ell$.

Remark 6.5. After $p$-completion, we obtain an $E_{\infty}$ structure on the $p$-completed connective Adams summand $\ell_{p}$. In subsequent work we have shown that this $E_{\infty}$ structure coincides with the one constructed by McClure and Staffeldt in [27] using algebraic $K$-theory.

\section{7. $E_{\infty}$ structures on $K O$}

The case of $K O$ can be treated by similar methods but involves somewhat more delicate considerations because of the presence of 2-torsion in $K O_{*}$. Recall that

$$
K O_{*}=\mathbb{Z}\left[h, y, w, w^{-1}\right] /\left(2 h, h^{3}, h y, y^{2}-4 w\right),
$$

where $h \in K O_{1}, y \in K O_{4}$ and $w \in K O_{8}$. We will also require the graded $\mathbb{Q}$-vector space $V_{*}=K O_{*} \otimes \widehat{\mathbb{Z}} / \mathbb{Z}$.

We will prove the following algebraic result.

\section{Theorem 7.1.}

(a) For any prime $p$ and $k \geqslant 1$, we have

$$
\mathrm{H} \Gamma^{*}\left(K O_{0} K O / p^{k} \mid \mathbb{Z} / p^{k} ; \mathbb{Z} / p^{k}\right)=0,
$$


and

$$
\mathscr{H} \Gamma^{*}\left(K O_{0} K \widehat{O_{p}} \mid \mathbb{Z}_{p} ; \mathbb{Z}_{p}\right)=\mathrm{H} \Gamma^{*}\left(K O_{0} K \widehat{O_{p}} \mid \mathbb{Z}_{p} ; \mathbb{Z}_{p}\right)=0
$$

(b) We have

$$
\mathrm{H} \Gamma^{n, *}\left(K O_{*} K O \mid K O_{*} ; K O_{*}\right)= \begin{cases}V_{*} & \text { if } n=1, \\ 0 & \text { otherwise }\end{cases}
$$

Using this, the obstruction theory $E_{\infty}$ structures and localization yield our result on $E_{\infty}$ structures for $K O$.

Theorem 7.2. $K O$ and, for each prime $p, K O_{(p)}$ and $K O_{p}$ all have unique $E_{\infty}$ structures.

To prove Theorem 7.1, we begin with a composite result distilled from [3] and $[1$, p. 162].

\section{Theorem 7.3.}

(a) $\mathrm{KO}_{*} \mathrm{KO}$ is a free $\mathrm{KO}_{*}$-module on countably many generators lying in $\mathrm{KO}_{0} \mathrm{KO}$.

(b) The natural homomorphism

$$
K \mathrm{O}_{0} \mathrm{KO} \longrightarrow \mathrm{KU} U_{0} \mathrm{KU} \stackrel{\cong}{\rightrightarrows} \mathrm{A}^{\mathrm{st}}
$$

is a split monomorphism whose image is

$$
\left\{f(w) \in \mathrm{A}^{\mathrm{st}}: f(-w)=f(w)\right\} \subseteq \mathrm{A}^{\mathrm{st}} .
$$

(c) For each prime $p$ and $k \geqslant 1$,

$$
K O_{0} K O / p^{k} \longrightarrow K U_{0} K U / p^{k} \cong \mathrm{A}^{\mathrm{st}} / p^{k}
$$

is a split monomorphism of $\mathbb{Z} / p^{k}$-modules.

Consider the short exact sequence

$$
0 \rightarrow K O_{*} \longrightarrow K O_{*} \otimes \widehat{\mathbb{Z}} \longrightarrow K O_{*} \otimes \widehat{\mathbb{Z}} / \mathbb{Z} \rightarrow 0 .
$$

Since $\widehat{\mathbb{Z}} / \mathbb{Z}$ is a $\mathbb{Q}$-vector space and we have the splitting of (5.1b), we can reformulate the above exact sequence to obtain that the sequence

$$
0 \rightarrow K O_{*} \longrightarrow \prod_{p} K O_{*} \otimes \mathbb{Z}_{p} \longrightarrow V_{*} \rightarrow 0
$$


is exact. Here $V_{*}$ is defined above and we have used the fact that each group $K O_{n}$ is finitely-generated. The application of $\Gamma$-cohomology of $K O_{*} K O$ to this sequence yields a long exact sequence which relates $\mathrm{H} \Gamma^{*, *}\left(K O_{*} K O \mid K O_{*} ; K O_{*}\right)$ to $\mathrm{H} \Gamma^{*, *}\left(K O_{*} K O \mid K O_{*} ; \prod_{p} K O_{*} \otimes \mathbb{Z}_{p}\right)$ and $\mathrm{H}^{*, *}\left(K O_{*} K O \mid K O_{*} ; V_{*}\right)$.

Now, for the part with coefficients in $V_{*}$ we have

$$
\mathrm{H} \Gamma^{*, *}\left(K O_{*} K O \mid K O_{*} ; V_{*}\right) \cong \mathrm{H} \Gamma^{*, *}\left(K O_{*} K O \otimes \mathbb{Q} \mid K O_{*} \otimes \mathbb{Q} ; V_{*}\right),
$$

and

$$
K O_{*} \otimes \mathbb{Q}=\mathbb{Q}\left[y, y^{-1}\right], \quad K O_{*} K O \otimes \mathbb{Q}=\mathbb{Q}\left[y, y^{-1}, z, z^{-1}\right] .
$$

By [29], as in Corollary 5.2 we find that

$$
\mathrm{H} \Gamma^{n, *}\left(K O_{*} K O \mid K O_{*} ; V_{*}\right)= \begin{cases}V_{*} & \text { if } n=1, \\ 0 & \text { otherwise. }\end{cases}
$$

For a fixed algebra, the cochain complex for $\Gamma$-cohomology commutes with limits taken over the coefficient module, therefore $\Gamma$-cohomology commutes with products of coefficient modules and the splitting $\widehat{\mathbb{Z}}=\prod_{p} \mathbb{Z}_{p}$ leads to

$$
\mathrm{H} \Gamma^{*, *}\left(K O_{*} K O \mid K O_{*} ; K O_{*} \otimes \widehat{\mathbb{Z}}\right) \cong \prod_{p} \mathrm{H} \Gamma^{*, *}\left(K O_{*} K O \mid K O_{*} ; K O_{*} \otimes \mathbb{Z}_{p}\right) .
$$

For each prime $p$ we obtain a short exact sequence,

$$
\begin{aligned}
0 \rightarrow \lim _{k}^{1} \mathrm{H} \Gamma^{*-1, *}\left(K O_{*} K O \mid K O_{*} ; K O_{*} / p^{k}\right) & \\
\longrightarrow \mathrm{H} \Gamma^{*, *} & \left(K O_{*} K O \mid K O_{*} ; K O_{*} \otimes \mathbb{Z}_{p}\right) \\
& \longrightarrow \lim _{k} \mathrm{H}^{*, *}\left(K O_{*} K O \mid K O_{*} ; K O_{*} / p^{k}\right) \rightarrow 0 .
\end{aligned}
$$

When $p>2$, we are reduced to considering

$$
\mathrm{H} \Gamma^{*, *}\left(K O_{*} K O \mid K O_{*} ; K O_{*} / p^{k}\right)=\mathrm{H}^{*, *}\left(K O_{*} K O / p^{k} \mid K O_{*} / p^{k} ; K O_{*} / p^{k}\right),
$$

which can be determined by the methods of Section 4 using the subgroup $\{ \pm 1\} \leqslant \mathbb{Z}_{p}^{\times}$ in place of the group of all $(p-1)$-st roots of unity. The result is that

$$
\mathrm{H} \Gamma^{*, *}\left(K O_{*} K O \mid K O_{*} ; K O_{*} / p^{k}\right)=0,
$$

whence

$$
\mathrm{H} \Gamma^{*, *}\left(K O_{*} K O \mid K O_{*} ; K O_{*} \otimes \mathbb{Z}_{p}\right)=0 .
$$

The case $p=2$ requires a more intricate analysis. First we identify $K O_{0} K O_{(2)}$ and the quotients $\mathrm{KO}_{0} \mathrm{KO} / 2^{k}$ as rings of functions. 


\section{Theorem 7.4.}

(a) There is an isomorphism of rings

$$
K O_{0} K O_{(2)} \cong\left\{f(w) \in \mathbb{Q}\left[w, w^{-1}\right]: f \mathbb{Z}_{(2)}^{\times} \subseteq \mathbb{Z}_{(2)}, f(-w)=f(w)\right\} \subseteq \mathrm{A}_{(2)}^{\text {st }} .
$$

(b) For each $k \geqslant 1$, there is an isomorphism of rings

$$
K O_{0} K O / 2^{k} \cong \operatorname{Cont}\left(1+8 \mathbb{Z}_{2}, \mathbb{Z} / 2^{k}\right),
$$

where $\operatorname{Cont}\left(1+8 \mathbb{Z}_{2}, \mathbb{Z} / 2^{k}\right)$ denotes the space of continuous maps from $1+8 \mathbb{Z}_{2} \subseteq \mathbb{Z}_{2}^{\times} \subseteq \mathbb{Z}_{2}$ with its 2 -adic topology to $\mathbb{Z} / 2^{k}$ with the discrete topology.

(c) There is an isomorphism of rings

$$
K O_{0} K \widehat{O_{2}} \cong \operatorname{Cont}\left(1+8 \mathbb{Z}_{2}, \mathbb{Z}_{2}\right)
$$

the space of continuous maps from $1+8 \mathbb{Z}_{2}$ to $\mathbb{Z}_{2}$.

(d) The algebras $\left(\mathrm{KO}_{0} \mathrm{KO} / 2^{k}, \mathbb{Z} / 2^{k}\right)$ and $\left(\mathrm{KO}_{0} \mathrm{KO}_{2}, \mathbb{Z}_{2}\right)$ are formally étale.

Proof. The methods of [4] apply here, and we leave verification of the details to the reader.

The squaring map $\mathbb{Z}_{2}^{\times} \longrightarrow \mathbb{Z}_{2}^{\times}$has image $1+8 \mathbb{Z}_{2}$, hence a polynomial $f(w) \in$ $\mathrm{A}_{(2)}^{\text {st }}$ satisfying $f(-w)=f(w)$ corresponds to a continuous function $1+8 \mathbb{Z}_{2} \longrightarrow \mathbb{Z}_{2}$. By compactness of the domain, $\operatorname{Cont}\left(1+8 \mathbb{Z}_{2}, \mathbb{Z} / 2^{k}\right)$ consists of locally constant functions. If we express $x \in \mathbb{Z}_{2}$ in the form

$$
x=x_{0}+x_{1} 2+x_{2} 2^{2}+\cdots+x_{n} 2^{n}+\cdots,
$$

where $x_{i}=0,1$, then the functions

$$
\xi_{i}: 1+8 \mathbb{Z}_{2} \longrightarrow \mathbb{Z}_{2} ; \quad \xi_{i}(x)=x_{i}
$$

are locally constant and give rise to $\mathbb{Z} / 2^{k}$-algebra generators of $\operatorname{Cont}\left(1+8 \mathbb{Z}_{2}, \mathbb{Z} / 2^{k}\right)$. They also satisfy the relations

$$
\xi_{i}^{2}=\xi_{i}
$$

and the distinct monomials

$$
\xi_{0}^{r_{0}} \xi_{1}^{r_{1}} \ldots \xi_{d}^{r_{d}} \quad\left(r_{i}=0,1\right)
$$

form a $\mathbb{Z} / 2^{k}$-basis. This implies that the $\mathbb{Z} / 2^{k}$-algebra $\operatorname{Cont}\left(1+8 \mathbb{Z}_{2}, \mathbb{Z} / 2^{k}\right)$ is formally étale. Similar considerations apply to the topological algebra $\mathrm{KO}_{0} \mathrm{KO}_{2}$. 
Collecting together the results of the above discussion (in particular Theorem 7.4(d)) we obtain the case $p=2$ of Theorem 7.1(a). The proof of Theorem 7.1(b) makes use of the long exact sequence resulting from (7.2) and (7.3).

We remark that rather than working modulo powers of 2 , it is also possible to consider powers of the maximal ideal $(2, h, y) \triangleleft K O_{*}$ and then we obtain

Proposition 7.5. For $k \geqslant 1$,

$$
\mathrm{H}^{*}\left(K O_{*} K O /(2, h, y)^{k} \mid K O_{*} /(2, h, y)^{k} ; K O_{*} /(2, h, y)^{k}\right)=0 .
$$

and

$$
\begin{aligned}
\mathscr{H} \Gamma^{*, *}\left(K O_{*} K \widehat{O_{(2, h, y)}} \mid\left(K O_{*} \widehat{)}_{(2, h, y)} ;\left(K O_{*} \widehat{(2, h, y)}\right)\right.\right. \\
=\mathrm{H} \Gamma^{*, *}\left(K O_{*} K \widehat{O_{(2, h, y)}} \mid\left(K O_{*} \widehat{)_{(2, h, y)}} ;\left(K O_{*}\right)_{(2, h, y)}\right)=0 .\right.
\end{aligned}
$$

\section{8. $E_{\infty}$ structures on the $I_{n}$-adic completion of $E(n)$}

In this section we describe what we can prove about $E_{\infty}$ structures on the $I_{n}$-adic completion of Johnson-Wilson spectrum $E(n)$ for a prime $p$ and $n \geqslant 1$.

The coefficient ring

$$
E(n)_{*}=\mathbb{Z}_{(p)}\left[v_{1}, \ldots, v_{n-1}, v_{n}, v_{n}^{-1}\right]
$$

is Noetherian and contains the maximal ideal

$$
I_{n}=\left(p, v_{1}, \ldots, v_{n-1}\right) \triangleleft E(n)_{*} .
$$

Here the $v_{i}$ denote the images of the Araki generators of $B P_{*}$ and we sometimes write $v_{0}=p$. There is a commutative ring spectrum $\widehat{E(n)}$ for which the coefficient ring $\widehat{E(n)}_{*}$ is the $I_{n}$-adic completion of $E(n)_{*}$, i.e., its completion at $I_{n}$. It is known from [13], [19] that $\widehat{E(n)}$ is the $K(n)$-localization of $E(n)$. We also know from [7] that for each prime $p, \widehat{E(n)}$ possesses a unique $A_{\infty}$ structure and the canonical map $\widehat{E(n)} \longrightarrow \widehat{E(n)} / I_{n} \simeq K(n)$ to the $n$-th Morava $K$-theory is a map of $A_{\infty}$ ring spectra for any of the $A_{\infty}$ structures on $K(n)$ shown to exist in [30]. Actually these results were only claimed for odd primes but the arguments also work for the prime 2 .

Proposition 8.1. Possible obstructions for an $E_{\infty}$ structure on the completed Johnson-Wilson spectra $\widehat{E(n)}$ live in the continuous $\Gamma$-cohomology groups

$$
\mathscr{H} \Gamma^{*, *}\left(\widehat{E(n)_{*}} \widehat{E(n)} \mid \widehat{E(n)}_{*} ; \widehat{E(n)_{*}}\right) .
$$


Proof. For $\widehat{E(n)}$ we have a continuous universal coefficient theorem, i.e., possible obstructions live in the continuous $\widehat{E(n)}$-cohomology of $\left(X_{m}\right)_{+} \ltimes_{\Sigma_{m}} E^{\wedge m}$, where $X_{m}$ is a filtration quotient of an $E_{\infty}$ operad as described in [31, section 5.1]. These cohomology groups can be identified with the continuous $\widehat{E(n)}{ }_{*}$-homomorphisms from the corresponding $\widehat{E(n)}$-homology groups (compare [31, proposition 5.4] and $[7, \S 1])$. This proves the claim.

For each $\ell \geqslant 0$, Proposition 2.2 yields a short exact sequence

$$
\begin{aligned}
& 0 \rightarrow \lim _{k}{ }^{1} \mathrm{H} \Gamma^{\ell-1, *}\left(E(n)_{*} E(n) / I_{n}^{k} \mid E(n)_{*} / I_{n}^{k} ; E(n)_{*} / I_{n}^{k}\right) \\
& \longrightarrow \mathcal{H} \Gamma^{\ell, *}\left(\widehat{E(n)_{*}} \widehat{E(n)} \mid \widehat{E(n)_{*}} ; \widehat{E(n)_{*}}\right) \\
& \longrightarrow \lim _{k} \mathrm{H} \Gamma^{\ell, *}\left(E(n)_{*} E(n) / I_{n}^{k} \mid E(n)_{*} / I_{n}^{k} ; E(n)_{*} / I_{n}^{k}\right) \rightarrow 0 .
\end{aligned}
$$

Theorem 8.2. The $E(n)_{*} / I_{n}^{k}$-algebra $E(n)_{*} E(n) / I_{n}^{k}$ is formally étale. Hence the $\Gamma$-cohomology of $E(n)_{*} E(n) / I_{n}^{k}$ over $E(n)_{*} / I_{n}^{k}$ is trivial,

$$
\mathrm{H}^{*, *}\left(E(n)_{*} E(n) / I_{n}^{k} \mid E(n)_{*} / I_{n}^{k} ; \widehat{E(n)}{ }_{*} / I_{n}^{k}\right)=0 .
$$

Proof. First we show that the algebra $E(n)_{*} E(n) / I_{n}^{k}$ is formally étale. In the following we use the notation chosen in [7]. As in the proof of [7, lemma 3.4], we can apply the infinite-dimensional Hensel lemma (see the proof of our Corollary 3.4) to split $E(n)_{*} E(n) / I_{n}^{k}$ into an infinite tensor product of $E(n)_{*} / I_{n}^{k}$-algebras,

$$
E(n)_{*} E(n) / I_{n}^{k}=\bigotimes_{j \geqslant 1} E(n)_{*} / I_{n}^{k}\left[S_{j}\right] /\left(v_{n} S_{j}^{p^{n}}-v_{n}^{p^{j}} S_{j}\right) .
$$

We can write $E(n)_{*} E(n) / I_{n}^{k}$ as a colimit of finite tensor products,

$$
E(n)_{*} E(n) / I_{n}^{k}=\operatorname{colim}_{m} \bigotimes_{j=1}^{m} E(n)_{*} / I_{n}^{k}\left[S_{j}\right] /\left(v_{n} S_{j}^{p^{n}}-v_{n}^{p^{j}} S_{j}\right) .
$$

We claim that each algebra $E(n)_{*} / I_{n}^{k}\left[S_{j}\right] /\left(v_{n} S_{j}^{p^{n}}-v_{n}^{p^{j}} S_{j}\right)$ is étale over $E(n)_{*} / I_{n}^{k}$. Notice that it is flat over $E(n)_{*} / I_{n}^{k}$ and is finitely-generated by $S_{j}$. As the ground ring $E(n)_{*} / I_{n}^{k}$ is Noetherian, the only thing that remains to be shown is that the module of Kähler differentials is trivial.

The Kähler differentials are generated by the symbol $d S_{j}$, but in $E(n)_{*} E(n) / I_{n}^{k}$ we have the relation $v_{n} S_{j}^{p^{n}}=v_{n}^{p^{j}} S_{j}$. The residue class of the element $v_{n} \in E(n)_{*}$ is a unit in the ring $E(n)_{*} / I_{n}$ and thus we can deduce

$$
d S_{j}=v_{n}^{1-p^{j}} d\left(S_{j}^{p^{n}}\right)=p^{n} v_{n}^{1-p^{j}} S_{j}^{p^{n}-1} d S_{j} .
$$


Iteration of this relation $t$ times, where $t$ is an integer such that $t n \geqslant k$, implies that $d S_{j}$ is zero, since in the quotient $E(n)_{*} / I_{n}^{k}, p^{k}$ is zero.

Now by Lemma $2.6 \Gamma$-homology commutes with colimits, therefore

$$
\begin{aligned}
\left.\mathrm{H} \Gamma_{*, *}\left(E(n)_{*} E(n) / I_{n}^{k} \mid E(n)_{*} / I_{n}^{k} ; \widehat{E(n)}\right)_{*} / I_{n}^{k}\right)=0 \\
\quad=\mathrm{H}^{*, *}\left(E(n)_{*} E(n) / I_{n}^{k} \mid E(n)_{*} / I_{n}^{k} ; \widehat{E(n)_{*}} / I_{n}^{k}\right) .
\end{aligned}
$$

This completes the proof of Theorem 8.2.

Using (8.1) and the fact that the completion of $\widehat{E(n)} \widehat{E(n)}$ is free on a countable basis [9, theorem 1.1], [4], we obtain

Theorem 8.3. For $p$ a prime and $n \geqslant 1$, the spectrum $\widehat{E(n)}$ possesses a unique $E_{\infty}$ structure.

Using the ideas of Section 2, we can also deduce

Theorem 8.4. For $n \geqslant 1$ and $k \geqslant 1$, we have

$$
\begin{aligned}
\mathrm{AQ}_{*}\left(E(n)_{*} E(n) / I_{n}^{k} \mid E(n)_{*} / I_{n}^{k} ; E(n)_{*} / I_{n}^{k}\right)=0 \\
=\mathrm{AQ}^{*}\left(E(n)_{*} E(n) / I_{n}^{k} \mid E(n)_{*} / I_{n}^{k} ; E(n)_{*} / I_{n}^{k}\right), \\
\mathrm{AQ}_{*}\left(E(n)_{*} E(n)_{I_{n}} \mid \widehat{E(n)}_{*} ; \widehat{E(n)}_{*}\right)=0 \\
=\mathrm{AQ}^{*}\left(E(n)_{*} E(n)_{I_{n}} \mid \widehat{E(n)_{*}} ; \widehat{E(n)_{*}}\right) .
\end{aligned}
$$

Remark 8.5. Extending Theorem 8.3 to cover $E(n)$ for $n>1$ does not appear to be straightforward. The following two problems arise.

- There is the question of whether $E(n)_{*} E(n)$ is a free $E(n)_{*}$-module when $n>1$. If $E(n)$ does not have a universal coefficient theorem, then the obstructions to building an $E_{\infty}$ structure on $E(n)$ would live in $E(n)$-cohomology which might not then be identifiable with $\Gamma$-cohomology. In [11], the first author showed that the cooperation algebra of the $I_{n}$-localization of $E(n), E(n)_{I_{n}}$, is free over $E(n)_{* I_{n}}$, so it does have a universal coefficient theorem and the above problem is overcome.

- $\Gamma$-cohomology of $E(n)_{*} E(n)$ is non-trivial in positive degrees. Even for $n=2$ there are polynomial generators in $E(2)_{*} E(2)$ which lead to non-trivial classes in $\Gamma$-cohomology.

We aim to return to the existence of $E_{\infty}$ structures on $E(n)$ and $E(n)_{I_{n}}$ in future work. 
We end this section with some remarks on suitably completed versions of elliptic cohomology. Here \&ll denotes the level 1 version of elliptic cohomology of Landweber, Ravenel and Stong [24] and we focus on the supersingular completions of [6]. Our above techniques together with results from [8] yield the following.

Theorem 8.6. For each prime $p>3$, the supersingular completions $\varepsilon \ell_{\left(p, E_{p-1}\right)}$ and El $\widehat{\mathcal{P}}$ for each maximal ideal $\mathcal{P} \triangleleft\left(\mathcal{E} \ell_{*}\right)_{(p)}$ containing $\left(p, E_{p-1}\right)$, have unique $E_{\infty}$ structures.

An analogous result applies to the $K(1)$-localization of $E \ell$ studied in [5] and more recently by M. Hopkins.

\section{An obstruction theory for the coherence of maps}

For the following, we need to work in a good category of spectra with a symmetric monoidal smash product, for example that of [17]. Where necessary, all ring spectra will be assumed to be fibrant.

Let $E$ and $F$ be two $E_{\infty}$ ring spectra over the $E_{\infty}$ operad $\mathcal{T}$ from [31, section 5.1] and let $f: E \longrightarrow F$ be a map of commutative ring spectra, i.e., the map $f$ commutes with the multiplication maps $\mu_{E}$ and $\mu_{F}$ up to homotopy,

$$
\mu_{F} \circ f \wedge f \simeq f \circ \mu_{E},
$$

and similar coherence properties exist with respect to the homotopies for associativity and commutativity on $E$ and $F$. The aim of the following discussion is to give criteria, when the map $f$ can be made into a map which is compatible with the $\mathcal{T}$-algebra structures on $E$ and $F$ up to homotopy. For $A_{\infty}$ structures the analogous question was addressed in [30].

From now on we will use the notation of [31]. The topological operad $\mathcal{T}$ is filtered by subspaces $\nabla^{m} \mathcal{T}(n) \subseteq \mathcal{T}(n)$. Let $\theta_{E}$ and $\theta_{F}$ be the action maps of the operad $\mathcal{T}$ on $E$ and $F$.

Consider the sequence of topological spaces

$$
\nabla^{m} \mathcal{T}(n) \hookrightarrow \nabla^{m+1} \mathcal{T}(n) \longrightarrow \nabla^{m+1} \mathcal{T}(n) / \nabla^{m} \mathcal{T}(n) \cup \partial \nabla^{m+1} \mathcal{T}(n),
$$

where $\partial \nabla^{m+1} \mathcal{T}(n)$ is the part of $\mathcal{T}(n)$ which is determined by compositions in the operad of elements coming from lower filtration degrees.

\section{Theorem 9.1.}

(a) If $\mathrm{H} \Gamma^{n, 2-n}\left(F_{*} E \mid F_{*} ; F_{*}\right)=0$ for all $n \geqslant 3$, then $f$ can be turned into a map satisfying

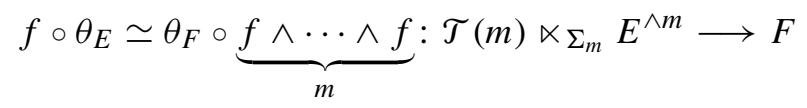

for all $m$. 
(b) If in addition $\operatorname{Hom}_{F_{*}}\left(F_{*} E, F_{*}\right) \cong \operatorname{Hom}_{E_{*}}\left(E_{*} E, F_{*}\right)$, then it suffices to prove that

$$
\mathrm{H} \Gamma^{n, 2-n}\left(E_{*} E \mid E_{*} ; F_{*}\right)=0
$$

for all $n \geqslant 3$.

The second condition is satisfied for instance if $F$ is projective over $E$, then $F_{*} E \cong F_{*} \otimes_{E_{*}} E_{*} E$ can be used to reduce the module of $F_{*}$-linear morphisms to the module of $E_{*}$-linear morphisms.

Proof. Assume $f$ satisfies the conditions up to filtration degree $m$. In order to extend $f$ coherently over the $(m+1)$-st filtration step, we have to show that the condition of the theorem suffices to force $f$ to fulfil

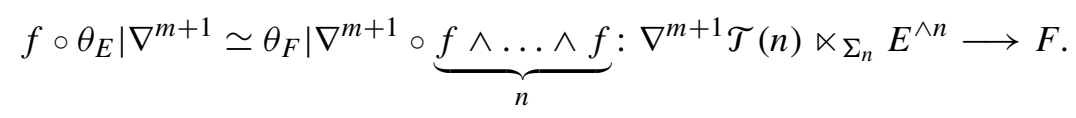

The map $f \circ \theta_{E} \mid \nabla^{m+1}$ corresponds to an element in $F^{0}\left(\nabla^{m+1} \mathcal{T}(n) \ltimes \Sigma_{n} E^{\wedge n}\right)$. Using the long exact cohomology sequence corresponding to the sequence of spaces

$$
\nabla^{m} \mathcal{T}(n) \cup \partial \nabla^{m+1} \mathcal{T}(n) \stackrel{i}{\rightarrow} \nabla^{m+1} \mathcal{T}(n) \stackrel{j}{\rightarrow} \nabla^{m+1} \mathcal{T}(n) / \nabla^{m} \mathcal{T}(n) \cup \partial \nabla^{m+1} \mathcal{T}(n),
$$

we find that the difference element

$$
f \circ \theta_{E}\left|\nabla^{m+1}-\theta_{F} \circ f^{\wedge n}\right| \nabla^{m+1}
$$

maps to zero under $i^{*}$, thus it has to be in the image of $j^{*}$. Consequently, if $j^{*}$ has trivial codomain, then this difference has to be trivial as an element in $F$-cohomology. An argument showing that the corresponding class in

$$
F^{0}\left(\nabla^{m+1} \mathcal{T}(n) / \nabla^{m} \mathcal{T}(n) \cup \partial \nabla^{m+1} \mathcal{T}(n) \ltimes \Sigma_{n} E^{\wedge n}\right)
$$

has to be a cocycle in the complex for $\mathrm{H} \Gamma^{*}$ can be found in [31]. Therefore, if $\mathrm{H} \Gamma^{m, 2-m}\left(F_{*} E \mid F_{*} ; F_{*}\right)$ vanishes in all degrees $m \geqslant 3$, the potentially obstructing difference maps $f \circ \theta_{E}-\theta_{F} \circ f^{\wedge n}$ have to be nullhomotopic.

From the triviality of $\mathrm{H} \Gamma^{n}$ when $n>1$ for complex $K$-theory and its localization at a prime $p$, we can deduce the following result.

Theorem 9.2. For each $k$ integer prime to $p$, the $k$-th Adams operation $\psi^{k}: K U_{(p)} \longrightarrow$ $K U_{(p)}$ can be refined to a coherent map with respect to the $E_{\infty}$ structure given by the operad action of $\mathcal{T}$ on $K U_{(p)}$. 
Proof. The action of such an Adams operation $\psi^{k}$ on $K U_{(p)_{2 n}} \cong \mathbb{Z}_{(p)}$ is given by multiplication by $k^{n}$, thus it induces a different $K U_{(p)_{*}} K U_{(p)}$-module structure on $K U_{(p)_{*}}$. This corresponds to taking $E=K U_{(p)}=F$ and the map $\psi^{k}: E \longrightarrow F$, then applying Theorem 9.1(b) and using the fact that the relevant $\Gamma$-cohomology groups vanish, this being a generalization of Theorem 6.2 which is proved in a similar way (this result depends crucially on the vanishing of $\Gamma$-cohomology for formally étale extensions).

Finally we have a result on the inclusion $j: E(1) \longrightarrow K U_{(p)}$ of the Adams summand into $p$-local $K$-theory which is a map of ring spectra.

Proposition 9.3. $j$ gives rise to a coherent map of $E_{\infty}$ spectra.

Proof. Using the Conner-Floyd isomorphism and the Landweber exactness of $E(1)$, the above argument can be adapted to prove that the relevant part of $\mathrm{H} \Gamma^{*, *}\left(K U_{(p)_{*}}(E(1)) \mid K U_{(p)_{*}} ; K U_{(p)_{*}}\right)$ vanishes.

Remark 9.4. With the aid of more machinery one can actually take the above arguments to obtain the existence of strict maps of $E_{\infty}$ ring spectra. Using a comparison result of Basterra and the second author [14, theorem 2.6], we can identify $\Gamma$-cohomology groups with the obstruction groups arising in the work of Goerss and Hopkins [18]. Now the Goerss-Hopkins obstruction theory [18, §4] tells us that the vanishing of the $\Gamma$-cohomology groups $\mathrm{H} \Gamma^{*, *}\left(K U_{(p)_{*}}(E(1)) \mid K U_{(p)_{*}} ; K U_{(p)_{*}}\right)$ and $\mathrm{H} \Gamma^{*, *}\left(K U_{(p)_{*}}\left(K U_{(p)}\right) \mid K U_{(p)_{*}} ; K U_{\left.(p)_{*}\right)}\right)$ implies that the Adams operations and the map $j$ give rise to maps of $E_{\infty}$ ring spectra.

\section{References}

[1] J. F. Adams, Infinite Loop Spaces. Ann. of Math. Stud. 90, Princeton University Press, Princeton, NY, 1978. Zbl 0398.55008 MR 0505692

[2] J. F. Adams and F. W. Clarke, Stable operations on complex $K$-theory. Illinois J. Math. 21 (1977), 826-829. Zbl 0379.55012 MR 0454977

[3] J.F. Adams, A. S. Harris and R. M. Switzer, Hopf algebras of co-operations for real and complex K-theory. Proc. Lond. Math. Soc. 23 (1971), 385-408. Zbl 0233.55014 MR 0293617

[4] A. Baker, $p$-adic continuous functions on rings of integers. J. London Math. Soc. 33 (1986), 414-420. Zbl 0562.12016 MR 0850957

[5] - Elliptic cohomology, $p$-adic modular forms and Atkin's operator $\mathrm{U}_{p}$. In Algebraic topology (Evanston, IL, 1988), M. Mahowald and S. Priddy (eds.), Contemp. Math. 96, Amer. Math. Soc., Providence, RI, 1989, 33-38. Zbl 0688.55006 MR 1022672

[6] -, On the homotopy type of the spectrum representing elliptic cohomology. Proc. Amer. Math. Soc. 107 (1989), 537-548. Zbl 0677.55005 MR 0982399 
Vol. 80 (2005) $\quad \Gamma$-cohomology of rings of numerical polynomials and $E_{\infty}$ structures 721

[7] -, $A_{\infty}$ structures on some spectra related to Morava $K$-theory. Quart. J. Math. Oxford (2) 42 (1991), 403-419. Zbl 0772.55003 MR 1135302

[8] - Operations and cooperations in elliptic cohomology. Part I: Generalized modular forms and the cooperation algebra. New York J. Math. 1 (1995), 39-74. Zbl 0810.55007 MR 1307488

[9] - A version of Landweber's filtration theorem for $v_{n}$-periodic Hopf algebroids. Osaka J. Math. 32 (1995), 689-699. Zbl 0868.55002 MR 1367899

[10] —, On the cohomology of some Hopf algebroids and Hattori-Stong theorems. Homology Homotopy Appl. 2 (2000), 29-40 (electronic). Zbl 0972.55003 MR 1750380

[11] —, $I_{n}$-local Johnson-Wilson spectra and their Hopf algebroids. Documenta Math. 5 (2000), 351-364. Zbl 0946.55002 MR 1767568

[12] A. Baker, F. Clarke, N. Ray and L. Schwartz, On the Kummer congruences and the stable homotopy of BU. Trans. Amer. Math. Soc. 316 (1989), 385-432. Zbl 0709.55012 MR 0942424

[13] A. Baker and U. Würgler, Liftings of formal group laws and the Artinian completion of $v_{n}^{-1}$ B P. Proc. Cambridge Philos. Soc. 106 (1989), 511-530. Zbl 0729.55002 MR 1010375

[14] M. Basterra and B. Richter, (Co-)homology theories for commutative ( $S$-)algebras. In Structured Ring Spectra, A. Baker and B. Richter (eds.), London Math. Soc. Lecture Notes Series 315, Cambridge University Press, Cambridge 2004, 115-131. Zbl 02152590 MR 2122156

[15] N. Bourbaki, Elements of Mathematics: Commutative Algebra. Chapters 1-7. Reprint of the 1989 English translation, Springer-Verlag, Berlin 1998. Zbl 0902.13001 MR 1727221

[16] F. Clarke, M. D. Crossley and S. Whitehouse, Bases for cooperations in $K$-theory. $K$-theory 23 (2001), 237-250. Zbl 0997.55021 MR 1857208

[17] A. Elmendorf, I. Kriz, M. Mandell and J. P. May, Rings, modules, and algebras in stable homotopy theory. Math. Surveys Monogr. 47, Amer. Math. Soc., Providence, RI, 1997. Zbl 0894.55001 MR 1417719

[18] P. G. Goerss and M. J. Hopkins, Moduli spaces of commutative ring spectra. In Structured Ring Spectra, A. Baker and B. Richter (eds.), London Math. Soc. Lecture Notes Series 315, Cambridge University Press, Cambridge 2004, 151-200. Zbl 02152592 MR 2125040

[19] M. Hovey and N. P. Strickland, Morava $K$-theories and localisations. Mem. Amer. Math. Soc. 139 (1999), no. 666. Zbl 0929.55010 MR 1601906

[20] C. U. Jensen, On the vanishing of $\lim ^{(i)}$. J. Algebra 15 (1970), 151-166. Zbl 0199.36202 MR 0260839

[21] —, Les foncteurs dérivés de lim et leurs applications en théorie des modules. Lecture Notes in Math. 254, Springer-Verlag, Berlin, New York 1972. Zbl 0238.18007 MR 0407091

[22] M. Joachim, A symmetric ring spectrum representing KO-theory. Topology 40 (2001), 299-308. Zbl 0979.55002 MR 1808222

[23] K. Johnson, The action of the stable operations of complex $K$-theory on coefficient groups. Illinois J. Math. 28 (1984), 57-63. Zbl 0555.55017 MR 0730711 
[24] P. S. Landweber, D. C. Ravenel and R. E. Stong, Periodic cohomology theories defined by elliptic curves. In The Čech centennial (Boston, MA, 1993), M. Cenkl et al. (eds.), Contemp. Math. 181, Amer. Math. Soc., Providence, RI, 1995, 317-337. Zbl 0920.55005 MR 1320998

[25] J.-L. Loday, Cyclic Homology. Second edition, Grundlehren Math. Wiss. 301, SpringerVerlag, Berlin 1998. Zbl 0885.18007 MR 1600246

[26] J. P. May, $E_{\infty}$ ring spaces and $E_{\infty}$ ring spectra. With contributions by F. Quinn, N. Ray and J. Tornehave, Lecture Notes in Math. 577, Springer-Verlag, Berlin, New York 1977. Zbl 0345.55007 MR 0494077

[27] J. E. McClure and R. E. Staffeldt, On the topological Hochschild homology of bu, I. Amer. J. Math. 115 (1993), 1-45. Zbl 0770.55010 MR 1209233

[28] C. Rezk, Notes on the Hopkins-Miller theorem. In Homotopy theory via algebraic geometry and group representations (Evanston, IL, 1997), M. Mahowald et al. (eds.), Contemp. Math. 220, Amer. Math. Soc., Providence, RI, 1998, 313-366. Zbl 0910.55004 MR 1642902

[29] B. Richter and A. Robinson, Gamma-homology of group algebras and of polynomial algebras. In Homotopy theory: relations with algebraic geometry, group cohomology, and algebraic K-theory, P. Goerss and S. Priddy (eds.), Contemp. Math. 346, Amer. Math. Soc., Providence, RI, 2004, 453-461. Zbl 02144717 MR 2066509

[30] A. Robinson, Obstruction theory and the strict associativity of Morava $K$-theories. In Advances in Homotopy Theory, B. Steer and W. Sutherland (eds.), London Math. Soc. Lecture Note Ser. 139, Cambridge University Press, Cambridge 1989, 143-152. Zbl 0688.55008 MR 1055874

[31] —, Gamma homology, Lie representations and $E_{\infty}$ multiplications. Invent. Math 152 (2003), 331-348. Zbl 1027.55010 MR 1974890

[32] - Classical obstructions and S-algebras. In Structured Ring Spectra, A. Baker and B. Richter (eds.), London Math. Soc. Lecture Notes Series 315, Cambridge University Press, Cambridge 2004, 133-49. Zbl 02152591 MR 2122157

[33] A. Robinson and S. Whitehouse, Operads and $\Gamma$-homology of commutative rings. Math. Proc. Cambridge Philos. Soc. 132 (2002), 197-234. Zbl 0997.18004 MR 1874215

[34] R. Schwänzl, R. M. Vogt and F. Waldhausen, Adjoining roots of unity to $E_{\infty}$ ring spectra in good cases - a remark. In Homotopy invariant algebraic structures (Baltimore, MD, 1998), J.-P. Meyer et al. (eds.), Contemp. Math. 239, Amer. Math. Soc., Providence, RI, 1999, 245-249. Zbl 0944.55006 MR 1718085

[35] S. Shatz, Profinite Groups, Arithmetic and Geometry. Ann. of Math. Stud. 67, Princeton University Press, Princeton, NJ, 1972. Zbl 0236.12002 MR 0347778

[36] C. A. Weibel, An Introduction to Homological Algebra. Cambridge Stud. Adv. Math. 38, Cambridge University Press, Cambridge 1994. Zbl 0797.18001 MR 1269324

[37] D. Zelinsky, Linearly compact modules and rings. Amer. J. Math. 75 (1953), 79-90. Zbl 0050.10802 MR 0051832 
Vol. 80 (2005) $\quad \Gamma$-cohomology of rings of numerical polynomials and $E_{\infty}$ structures

Received May 12, 2003

Andrew Baker, Mathematics Department, University of Glasgow, Glasgow G12 8QW, Scotland

E-mail: a.baker@maths.gla.ac.uk

URL: http://www.maths.gla.ac.uk/ ajb

Birgit Richter, Fachbereich Mathematik der Universität Hamburg, Bundesstrasse 55, 20146 Hamburg, Germany

E-mail: richter@math.uni-hamburg.de

URL: http://www.math.uni-hamburg.de/home/richter/ 